\title{
Dynamical Entropy of Space Translations of CAR and CCR Algebras with respect to Quasi-free States
}

\author{
Yong Moon Park ${ }^{1 \star}$ and Hyun Hye Shin ${ }^{2 \star \star}$ \\ 1 Department of Mathematics, Yonsei University, Seoul 120-749, Korea \\ 2 Department of Mathematics, Seoul National University, Seoul 151-712, Korea
}

Received April 7, 1992; in revised form September 17, 1992

\begin{abstract}
We compute the dynamical entropy in the sense of Connes, Narnhofer and Thirring of space translations of the CAR and CCR algebras in $\nu$-dimensional continuous spaces with respect to invariant quasi-free states. It turns out that the dynamical entropies are equal to the corresponding mean entropies of the systems under consideration. Computing the mean entropies explicitly we derive the entropy formulas for the systems.
\end{abstract}

\section{Introduction}

In their recent paper [7] Connes, Narnhofer and Thirring extended the notion of a dynamical entropy of classical dynamical systems introduced by Kolmogorov and Sinai $[9,18]$ to the case of automorphisms of $C^{*}$-algebras invariant with respect to a given state. The dynamical entropy is the maximal entropy increase of a subalgebra per unit time and measure how chaotically the system evolves. As in the classical ergodic theory [5], the concept of the entropy should be mathematically useful to find a classification of quantum chaotic evolutions. Some attempts have been undertaken in this direction $[10,12,13]$.

In order to find a classification of automorphisms of $C^{*}$-algebras, it should be important to develop the methods which enable to compute the entropy for quantum systems. There have been some results in this field. The dynamical entropies of space translation for the Gibbs state of one dimensional bounded quantum lattice systems $[7,11]$ and the quasi-free evolutions of the CAR algebras [19] have been computed by utilizing continuity properties of the entropy [7, 19]. Recently the chaotic behavior of automorphisms on the rotation algebra [10] and noncommutative 2-shift [13] were investigated. In [16] we have extended the continuity [7] of the Kolmogorov-Sinai type for AF-algebras to non-AF situations and applied it to the unbounded quantum spin

\footnotetext{
$\star$ Research supported in part by the Basic Science Research Program, Ministry of Education, 1992

$\star \star$ Research supported in part by GARC in 1991-1992
} 
systems with product states. In this paper we shall compute the dynamical entropy of space translations of the CAR and CCR algebras in $\nu$-dimensional continuous spaces with respect to invariant quasi-free states. The main methods we use will be a further extension of the entropy results in [16] and a careful investigation of the entropy defects.

We shall work within the framework of quantum statistical mechanical systems where we start from strictly local algebra $\mathscr{C}_{\Lambda}$ indexed by bounded regions $\Lambda$ in $\mathbb{R}^{\nu}$. The norm closure of $\bigcup_{\Lambda} \mathscr{A}_{\Lambda}$ is the $C^{*}$-algebra $\mathscr{A}$ of quasi-local operators. For each bounded region $\Lambda \subset \mathbb{R}^{\nu}$, let $\widehat{A}_{\Lambda}$ be the CAR algebra (resp. the CCR algebra) of local observables satisfying the canonical anticommutation relations (resp. the canonical commutation relations). Let $K: \mathbb{R}^{\nu} \rightarrow \mathbb{R}$ be an integrable function and let $\hat{K}$ be its Fourier transform:

$$
\hat{K}(k)=\int K(x) e^{-\imath k \cdot x} d^{\nu} x,
$$

where $k \cdot x=\sum_{i=1}^{\nu} k_{i} x_{i}$. We assume that $0 \leq \hat{K}(k) \leq 1$ for the CAR algebra and $0 \leq \hat{K}(k) \leq M<\infty$ for the CCR algebra, respectively. Let $A$ be the bounded operator on $\bar{L}^{2}\left(\mathbb{R}^{\nu}\right)$ given by

$$
(A f)(x)=\int K(x-y) f(y) d^{\nu} y .
$$

Then the gauge invariant quasi-free state $\omega_{A}$ on $\mathscr{A}$ is translational invariant. For more details, see Sect. 3. Denote by $\theta$ the representation of $\mathbb{Z}^{\nu}$-action on $\mathscr{A}$. Then $\left(\mathscr{b}, \theta, \omega_{A}\right)$ is the $C^{*}$-dynamical system we are considering. Let $h_{\omega_{A}}(\theta)$ be the dynamical entropy of $\theta$ with respect to $\omega_{A}$ [7]. Let $\eta$ denote the real function on $[0, \infty)$ defined by $\eta(0)=0, \eta(t)=-t \log t$ for $t \in(0, \infty)$. We shall prove the formulas

$$
h_{\omega_{A}}(\theta)=\frac{1}{(2 \pi)^{\nu}} \int\{\eta(\hat{K}(k))+\eta(1-\hat{K}(k))\} d^{\nu} k \quad(\mathrm{CAR})
$$

and

$$
h_{\omega_{A}}(\theta)=\frac{1}{(2 \pi)^{\nu}} \int\{\eta(\hat{K}(k))-\eta(1+\hat{K}(k))\} d^{\nu} k \quad(\mathrm{CCR})
$$

under appropriate conditions on $A$. For more details, see Sect. 3 .

It may be worth to give some comments on the dynamical systems we are dealing with. Our result in this paper can be viewed as the first step in the study of the dynamical properties of interacting particle systems in quantum statistical mechanics in continuous spaces $[14,17]$. The main difficulty involved in the derivation of the formulas (1.1) and (1.2) is the fact that each local algebra $\mathscr{A}_{\Lambda}$ is infinite dimensional, which is contrasted to the bounded quantum lattice systems studied in [7, 11]. In [19] Störmer and Voiculescu derived a formula similar to that in (1.1) for the entropy of Bogoliubov transformations of the CAR algebra when the operator $A$ in the quasifree state $\omega_{A}$ has pure point spectrum. But in our case the operator $A$ has continuous spectrum and so our result for the CAR algebra may be considered as a complement to that in [19]. Because of the reasons stated above, the derivation of the formulas in (1.1) and (1.2) turns out to be technically more complicated than the previous calculations $[6,7,10-13,19]$. 
We organize the paper as follows: In Sect.2, we recall the definition of the dynamical entropy of $C^{*}$-algebras in [7] and describe briefly an entropy result on quasi-local algebras in [16]. We then extend the result of [16] to the CAR algebras which will be needed in the sequel. In Sect.3, we review the notion of quasi-free states on CAR and CCR algebras. After listing the basic assumptions (Assumption 3.1) we state our main result (Theorem 3.3) which says that the dynamical entropies of the systems are equal to the corresponding mean entropies. The proof of the main result is divided into several parts. In Sect.4, we use the general entropy results in Sect. 2 to show that the dynamical entropies of the systems are bounded by the corresponding mean entropies. We then introduce a specific decomposition of the state $\omega_{A}$ and reduce the proof of the main result to that of vanishing of the mean entropy defect (Proposition 4.3). In Sect. 5 we collect basic apriori estimates. Sect. 6 is the heart of the paper where we show that the thermodynamic limit of the average of entropy defects vanishes, and so complete the proof of the main result. We compute the mean entropies of the systems explicitly in the Appendix, and derive the entropy formulas (1.1) and (1.2).

\section{Dynamical Entropy and Some General Results}

As a preparation, we review the definition of the dynamical entropy of $C^{*}$-algebras and then collect some entropy results which do not involve quasi-free states and which are needed in the sequel. Throughout this section we consider a $C^{*}$-dynamical system $(\mathscr{A}, \theta, \phi)$ where $\mathscr{A}$ is a unital $C^{*}$-algebra, $\theta$ is an automorphism on $\mathscr{A}$ and $\phi$ is a state over $\mathscr{A}$ which is invariant with respect to $\theta$.

Let $\mathscr{C}$ be a finite dimensional $C^{*}$-algebra and let $\phi$ and $\psi$ be states on $\mathscr{b}$. The density operators corresponding to $\phi$ and $\psi$ are denoted by $\varrho_{\phi}$ and $\varrho_{\psi}$, respectively. The relative entropy for the states $\phi$ and $\psi$ is defined by

$$
S(\phi \mid \psi) \equiv \operatorname{Tr}\left(\varrho_{\psi}\left(\log \varrho_{\psi}-\log \varrho_{\phi}\right)\right),
$$

where $\operatorname{Tr}$ denotes the trace.

We recall the definition of the CNT entropy in [7]. Let $\mathscr{t}$ be a unital $C^{*}$-algebra, $\mathscr{N}_{1}, \ldots, \mathscr{N}_{k}$ finite dimensional $C^{*}$-algebras and $\gamma_{\jmath}: \mathscr{N}_{j} \rightarrow \mathscr{A}_{6}$ a completely positive unital map, $j=1, \ldots, k$. Let $\phi$ be a state on $\mathscr{A}$ and $P: \mathscr{A} \rightarrow \mathscr{B}$ a completely positive unital map of $\mathscr{C}$ into a finite dimensional abelian $C^{*}$-algebra $\mathscr{B}$ such that there is a state $\mu$ on $\mathscr{B}$ for which $\mu \circ P=\phi$. If $p_{1}, \ldots, p_{r}$ are the minimal projections in $\mathscr{B}$, then there are states $\phi_{i}, i=1, \ldots, r$, of $\mathscr{A}$ such that

$$
P(x)=\sum_{i=1}^{r} \phi_{i}(x) p_{i}, \quad x \in \mathscr{b} .
$$

Since $\mu \circ P=\phi$,

$$
\phi=\sum_{i=1}^{r} \mu\left(p_{i}\right) \phi_{i} .
$$

That is, $\phi$ can be written as a convex combination of the $\phi_{i}$. As in [7], let

$$
\varepsilon_{\mu}(P)=\sum \mu\left(p_{i}\right) S\left(\phi \mid \phi_{\imath}\right) .
$$


The entropy defect is given by

$$
S_{\mu}(P)=S(\mu)-\varepsilon_{\mu}(P) .
$$

where $S(\mu)=-\sum_{i=1}^{r} \mu\left(p_{i}\right) \log \mu\left(p_{i}\right)$ is the entropy of $\mu$.

Let $\mathscr{B}, j=1, \ldots, k$, be $C^{*}$-subalgebras of $\mathscr{B}$ and let $E_{j}: \mathscr{B} \rightarrow \mathscr{S}_{j}$ a $\mu$-invariant conditional expectation. Then the quadruple $\left(\mathscr{\mathscr { S }}, E_{j}, P, \mu\right)$ is called an abelian model for $\left(\mathscr{A}, \phi, \gamma_{1}, \ldots, \gamma_{k}\right)$ and its entropy is defined to be

$$
S\left(\left.\mu\right|_{\underset{j}{\bigvee} \mathscr{B}_{j}}\right)-\sum_{j} S_{\mu}\left(\varrho_{j}\right)
$$

where $\varrho_{\jmath}=E_{j} \circ P \circ \gamma_{j}: \mathscr{N}_{j} \rightarrow \mathscr{S}_{j}$ is a completely positive map from $\mathscr{N}_{j}$ to $\mathscr{R}_{j}$. The supremum of the entropies of all such abelian models is denoted by

$$
H_{\phi}\left(\gamma_{1}, \ldots, \gamma_{k}\right) \text {. }
$$

If $\theta$ is $\phi$-invariant automorphism of $\mathscr{A}$, let $\gamma: \mathscr{V}^{\circ} \rightarrow \mathscr{A}$ be a completely positive unital map of a finite dimensional $C^{*}$-algebra $\mathscr{N}^{*}$ to $\mathscr{A}$, and denote by

$$
h_{\phi, \theta}(\gamma)=\lim _{k \rightarrow \infty} \frac{1}{k} H_{\phi}\left(\gamma, \theta \circ \gamma, \ldots, \theta^{k-1} \circ \gamma\right) .
$$

The dynamical entropy of $\theta$ with respect to $\phi$ is defined by

$$
h_{\phi}(\theta)=\sup _{\gamma} h_{\phi, \theta}(\gamma) \text {. }
$$

For the details, we refer the reader to [7].

Next we recall an entropy result on quasi-local algebras from [16] which will be applied to the CCR algebra. We work exclusively within the framework of quantum statistical mechanical systems where we start from strictly local algebras to $_{\Lambda}$ indexed by bounded regions $\Lambda$ in $\mathbb{R}^{\nu}$ (or $\mathbb{Z}^{\nu}$ ). The norm closure of $\bigcup_{\Lambda} \mathscr{A}_{\Lambda}$ is the $C^{*}$-algebra A of quasi-local operators. For the general definition of quasi-local algebras indexed by directed sets, we refer to [3]. Let . $t$ be a quasi-local algebra whose generating net $\left\{\mathscr{A}_{\Lambda}\right\}$ is formed of von Neumann algebras $\mathscr{C}_{\Lambda}$ in separable Hilbert spaces $\mathscr{H}_{\Lambda}$. A state $\phi$ on. $t_{0}$ is said to be locally normal if $\phi$ is normal in restriction to each $\mathscr{A}_{\Lambda}$. Then $\phi$ in restriction to each $\mathscr{l}_{\Lambda}$ is determined by a density matrix $\varrho_{\Lambda}$ on a Hilbert space $\mathscr{H}_{\Lambda}$. We list some assumptions on quasi-local algebras.

Assumption 2.1. Let $\left(A,\left\{A_{A}\right\}\right)$ be a quasi-local algebra indexed by bounded open regions $\Lambda \subset \mathbb{R}^{\nu}$ (or $\mathbb{Z}^{\nu}$ ) and let $\phi$ be a state on $\mathscr{G}$. We assume that the following properties are valid:

(a) For each $\Lambda, \mathscr{t}_{\Lambda}$ is a von Neumann algebra in a separable Hilbert space $\mathscr{H}_{\Lambda}$.

(b) Tensor product property: Let $\Lambda_{1} \subset \Lambda$. Then there exists $\Lambda_{2} \subset \Lambda$ such that $\Lambda_{1} \cap \Lambda_{2}=\emptyset$ and $\cdot A_{\Lambda}=A_{\Lambda_{1}} \otimes \mathscr{b}_{\Lambda_{2}}$.

(c) Locally normal property: $\phi$ is locally normal and for each $\Lambda$ the corresponding density matrix $\varrho_{\Lambda}$ belongs to $\mathscr{A}_{\Lambda}$.

For a given $\Lambda$, let $\lambda_{i}, i=1,2, \ldots$, be the eigenvalues of the density matrix $\varrho_{\Lambda}$ listed in decreasing order (counting multiplicities), and let $\mathscr{H}_{\Lambda, n}$ be the subspace 
of $\mathscr{H}_{\Lambda}$ spanned by the eigenvectors corresponding to eigenvalues $\lambda_{1}, \lambda_{2}, \ldots, \lambda_{n}$. Denote by $P_{n}$ the projection operator from $\mathscr{H}_{\Lambda}$ to $\mathscr{H}_{\Lambda, n}$ and

$$
\mathscr{A}_{\Lambda, n} \equiv P_{n} \mathscr{A}_{\Lambda} P_{n} \oplus \mathbb{C} \cdot P_{n}^{\perp}
$$

where $P_{n}^{\perp}=1-P_{n}$. Then from Assumption 2.1(c) it follows that each $\mathscr{C}_{\Lambda, n}$ is a finite dimensional unital subalgebra of $\mathscr{C}_{\Lambda}$. For each $\Lambda$ and $n$, let $\tau_{\Lambda, n}$ be the embedding map of $\mathscr{A}_{\Lambda, n}$ into $\mathscr{A}_{\Lambda}$. Throughout the paper we will adapt the convention that $\Lambda \uparrow \mathbb{R}^{\nu}$ indicates $\Lambda$ increases to $\mathbb{R}^{\nu}$ so that $\Lambda$ eventually contains any bounded region of $\mathbb{R}^{\nu}$. The following is the main result in [16]:

Theorem 2.2 [16, Theorem 3.2]. Let $\left(\mathscr{A},\left\{\mathscr{A}_{\Lambda}\right\}\right)$ be a quasi-local algebra indexed by the bounded regions $\Lambda \subset \mathbb{R}^{\nu}$ (or $\mathbb{Z}^{\nu}$ ), $\theta$ an automorphism on Ao and $\phi$ a state on . A invariant under $\theta$. Under the assumptions in Assumption 2.1, one has

$$
h_{\phi}(\theta)=\lim _{\Lambda \uparrow \mathbb{R}^{\nu}} \lim _{n \rightarrow \infty} h_{\phi, \theta}\left(\mathscr{C}_{\Lambda, n}\right) .
$$

Here the convention that the subalgebra $A_{\Lambda, n}$ is standing for the inclusion map $\mathscr{A}_{\Lambda, n} \rightarrow \mathscr{b}$ has been used.

Remark 2.3. (a) The tensor product in Assumption 2.1(b) is the von Neumann algebra tensor product [8].

(b) In the most quantum statistical mechanical systems the equilibrium states obtained via thermodynamic limits of local Gibbs states turn out to be locally normal $[3,14$, $15,17]$.

(c) There was a small gap in the proof of the above theorem in [16]. We shall discuss it precisely and fill up the gap at the end of this section. See Remark 2.5.

We shall apply Theorem 2.2 to get an upper bound of the entropy for the CCR algebra. In the case of the CAR algebra we do not have the tensor product property in Assumption 2.1(b). Thus in order to get the result analogous to Theorem 2.2 for the CAR algebra, we have to modify slightly the method used in the proof of Theorem 2.2 in [16]. For each $\Lambda \subset \mathbb{R}^{\nu}$, let $\mathscr{C}_{\Lambda}$ be the CAR algebra over $L^{2}(\Lambda)$. See Sect. 3 for the details. Then $\mathscr{A}_{\Lambda}$ is isomorphic to $\mathscr{L}\left(\mathscr{F}_{\Lambda}\right)$, where $\mathscr{F}_{\Lambda}$ is the (antisymmetric) Fock space [3]. Let $\mathscr{A}$ be the norm closure of $\bigcup_{\Lambda} \mathscr{A}_{\Lambda}$. Then $\left(\mathscr{A},\left\{\mathscr{C}_{\Lambda}\right\}\right)$ is a quasi-local algebra [3]. The following is an extension of Theorem 2.2 to the CAR algebra.

Theorem 2.4. Let $\left(\mathscr{A}_{,}\left\{\mathscr{A}_{\Lambda}\right\}\right)$ be the CAR quasi-local algebra indexed by bounded open region $\Lambda \subset \mathbb{R}^{\nu}, \theta$ an automorphism on $\mathscr{A}$ and $\phi$ a locally normal state on $\mathscr{A}$ invariant under $\theta$. Then one has

$$
h_{\phi}(\theta)=\lim _{\Lambda \uparrow \mathbb{R}^{\nu}} \lim _{n \rightarrow \infty} h_{\phi, \theta}\left(\mathcal{A}_{\Lambda, n}\right),
$$

where $\mathscr{A}_{\Lambda, n}$ is the subalgebra defined in (2.9).

Proof. If we are able to show that for each $\Lambda \subset \mathbb{R}^{\nu}$ there is a completely positive unital map $\sigma_{\Lambda}: \mathscr{b} \rightarrow \mathscr{b}_{\Lambda}$ such that

$$
\lim _{\Lambda \uparrow \mathbb{R}^{\nu}}\left\|\tau_{\Lambda} \circ \sigma_{\Lambda}(x)-x\right\|=0
$$

for each $x \in \mathscr{A}$ [16, Lemma 4.1], where $\tau_{\Lambda}: \mathscr{A}_{\Lambda} \rightarrow \mathscr{A}$ is the inclusion map, the proof of the theorem will be exactly the same as that of Theorem 2.2 in [16]. As in 
[16], we first define $\sigma_{\Lambda}$ on the norm dense subalgebra $\bigcup_{\Lambda^{\prime}} \mathscr{A}_{\Lambda^{\prime}}$ as follows: For each $\Lambda^{\prime}$ with $\Lambda \subset \Lambda^{\prime}$, let $\mathscr{F}_{\Lambda^{\prime}}$ be the family of finite linear combinations of elements in $\left\{y z \mid y \in \mathscr{A}_{\Lambda}, z \in \mathscr{A}_{\Lambda^{\prime \prime}}\right\}$, where $\Lambda^{\prime \prime}=\operatorname{int}\left(\Lambda^{\prime} \backslash \Lambda\right)$. Since $L^{2}\left(\Lambda^{\prime}\right)=L^{2}(\Lambda) \oplus L^{2}\left(\Lambda^{\prime \prime}\right)$, it follows that $\mathscr{S}_{\Lambda^{\prime}}$ is weakly dense in $\mathscr{A}_{\Lambda^{\prime}}, \mathscr{t}_{\Lambda^{\prime}}=\mathscr{L}\left(\mathscr{F}_{\Lambda^{\prime}}\right)$. Let $\Omega$ be the Fock vacuum vector [3]. For each $y \in \mathscr{A}_{\Lambda}$ and $z \in \mathscr{A}_{\Lambda^{\prime \prime}}$, define

$$
\sigma_{\Lambda}(y z)=(\Omega, z \Omega) y \text {. }
$$

Since $(\Omega, z \Omega)=0$ for each odd element $z \in \ell_{\Lambda^{\prime \prime}}$, it follows that $\sigma_{\Lambda}(y z)=\sigma_{\Lambda}(z y)$, and so $\sigma_{\Lambda}$ is well defined. By linearity, $\sigma_{\Lambda}$ extends to $\mathscr{S}_{\Lambda^{\prime}}$. An inspection shows that $\sigma_{\Lambda}\left(b_{1} a b_{2}\right)=b_{1} \sigma_{\Lambda}(a) b_{2}, b_{\imath} \in \mathscr{t}_{\Lambda}, a \in \mathscr{S}_{\Lambda^{\prime}}$. Thus $\sigma_{\Lambda}$ is a unital conditional expectation from $\mathscr{S}_{\Lambda^{\prime}}$ to $\mathscr{A}_{\Lambda}$, and so it is a completely positive unital map. Any vectors $\phi, \psi \in \mathscr{F}_{\Lambda}$ can be considered as vectors in $\mathscr{F}_{\Lambda^{\prime}}, \Lambda \subset \Lambda^{\prime}$. By (2.11) it is easy to check that

$$
\left(\phi, \sigma_{\Lambda}(x) \psi\right)=(\phi, x \psi), \quad x \in \mathscr{S}_{\Lambda^{\prime}} .
$$

Thus by a corollary of Hahn-Banach theorem it turns out that $\sigma_{\Lambda}$ is a contraction.

Next we extend $\sigma_{\Lambda}$ to $\mathscr{l}_{\Lambda^{\prime}}, \Lambda \subset \Lambda^{\prime}$. Let an element $x$ in $\mathscr{t}_{\Lambda^{\prime}}$ with unit norm $(\|x\|=1)$ be given, and let $\mathscr{B}_{1}$ be the closed unit ball in $\mathscr{C}_{\Lambda^{\prime}}$. Since $\mathscr{S}_{\Lambda^{\prime}} \cap \mathscr{B}_{1}$ is $\sigma$ strong* dense in $\mathscr{C}_{\Lambda^{\prime}} \cap \mathscr{P}_{1}$ by the Kaplansky denisty theorem, there exists a sequence $\left\{x_{n}\right\}$ in $\mathscr{S}_{\Lambda^{\prime}} \cap \mathscr{B}_{1}$ which converges to $x$ in the $\sigma$-strong* topology (and so in the weak topology). By (2.12), $\left\{\sigma_{\Lambda}\left(x_{n}\right)\right\}$ converges to an element $y \in \mathscr{A}_{\Lambda}$. Put $\sigma_{\Lambda}(x)=y$. Since $\sigma_{\Lambda}$ is a contraction on $\mathscr{S}_{\Lambda^{\prime}}$, it follows that $\left\|\sigma_{\Lambda}(x)\right\| \leq \varlimsup_{n \rightarrow \infty}\left\|\sigma_{\Lambda}\left(x_{n}\right)\right\| \leq 1$. Thus we have extended $\sigma_{\Lambda}$ to $\mathscr{C}_{\Lambda^{\prime}}$ as a contraction. By continuity $\sigma_{\Lambda}$ extends to $\mathscr{A}$.

We next show the convergence in (2.10). For any $x \in \mathscr{b}$ and $\varepsilon>0$, there exists $\Lambda^{\prime}$ and $x^{\prime} \in \mathscr{A}_{\Lambda^{\prime}}$ such that $\left\|x-x^{\prime}\right\|<\varepsilon / 2$. Notice that $\tau_{\Lambda} \circ \sigma_{\Lambda}\left(x^{\prime}\right)=x^{\prime}$ for $\Lambda^{\prime} \subset \Lambda$. For given $x \in \mathscr{A}_{b}$ and $\varepsilon>0$ we choose $\Lambda^{\prime}$ and $x^{\prime} \in \mathscr{A}_{\Lambda^{\prime}}$ as above. Then for any $\Lambda$ with $\Lambda^{\prime} \subset \Lambda$, it follows that

$$
\begin{aligned}
\left\|\tau_{\Lambda} \circ \sigma_{\Lambda}(x)-x\right\| & \leq\left\|\tau_{\Lambda} \circ \sigma_{\Lambda}\left(x-x^{\prime}\right)\right\|+\left\|x-x^{\prime}\right\| \\
& \leq 2\left\|x-x^{\prime}\right\|<\varepsilon .
\end{aligned}
$$

This proves the convergence in (2.10) completely. The remainder of the proof is exactly same as that of Theorem 2.2 in [16]. For details, we refer to [16]. Q.E.D.

Remark 2.5. As stated in Remark 2.3(c), there was a small gap in the proof of Lemma 4.1 in [16]. The lemma stated that under Assumption 2.1, there is a completely positive unital map $\sigma_{\Lambda}: \mathscr{A} \rightarrow \mathscr{A}_{\Lambda}$ such that the convergence in (2.10) holds. For $\Lambda \subset \Lambda^{\prime}$, let $\mathscr{S}_{\Lambda^{\prime}}$ be the family of finite linear combinations of elements in $\left\{y \otimes z \mid y \in \mathscr{t}_{\Lambda}, z \in \mathscr{b}_{\Lambda^{\prime \prime}}\right\}$. Since $\mathscr{t}_{\Lambda^{\prime}}=\mathscr{A}_{\Lambda} \otimes \mathscr{C}_{\Lambda^{\prime \prime}}, \mathscr{S}_{\Lambda^{\prime}}$ is weakly dense in $\mathscr{A}_{\Lambda^{\prime}}$. In [16], we defined $\sigma_{\Lambda}$ as

$$
\sigma_{\Lambda}(y \otimes z)=\phi(z) y, \quad y \in \mathscr{A}_{\Lambda}, z \in \mathscr{C}_{\Lambda^{\prime \prime}},
$$

where $\phi$ is a state on $\mathscr{A}$. The state $\phi$ should be the locally normal state in Assumption 2.1(a). Then the conclusion in [16, Lemma 4.1] holds by the following arguments: By linearity $\sigma_{\Lambda}$ extends to $\mathscr{S}_{\Lambda^{\prime}}$. Since $\phi$ is locally normal and so the restriction of $\phi$ to $\mathscr{C}_{\Lambda^{\prime \prime}},\left.\phi\right|_{\mathscr{A}_{\Lambda^{\prime \prime}}}$, is normal. For any $\psi_{1}, \psi_{2} \in \mathscr{H}_{\Lambda}$, let $\omega_{\psi_{1}, \psi_{2}}$ be the state on $\mathscr{A}_{\Lambda}$ defined by $\omega_{\psi_{1}, \psi_{2}}(x)=\left(\psi_{1}, x \psi_{2}\right), x \in \mathscr{A}_{\Lambda}$. Then $\left.\omega_{\psi_{1}, \psi_{2}} \otimes \phi\right|_{\ell_{\Lambda^{\prime \prime}}}$ is a normal state on $\mathscr{A}_{\Lambda^{\prime}}=\mathscr{A}_{\Lambda} \otimes \mathscr{A}_{\Lambda^{\prime \prime}}$. By using the Kaplansky density theorem and the argument used in the proof of Theorem $2.4, \sigma_{\Lambda}$ extends to $\mathscr{C}_{\Lambda^{\prime}}$ as an completely positive contractive unital map. This fills the gap in the proof of Lemma 4.1 in [16] completely. 


\section{Quasi-Free States on CAR and CCR Algebras and Main Result}

In this section we briefly review the notion of quasi-free states on CAR and CCR algebras, and then state our main result. For detailed descriptions on the CAR and CCR algebras, we refer the reader to $[2,3,20]$. Since these algebras are uniquely determined by the appropriate form of commutation relations [3], we shall deal with the CAR and CCR algebras in the Fock spaces.

Let $\mathscr{F}_{+}$(resp. $\mathscr{F}_{-}$) be the symmetric (resp. antisymmetric) Fock space over $L^{2}\left(\mathbb{R}^{\nu}\right)$. For each bounded region $\Lambda \subset \mathbb{R}^{\nu}$, let $\mathscr{F}_{\Lambda, \pm}$ be the corresponding Fock space over $L^{2}(\Lambda)$. Denote by $a_{ \pm}(f)$ and $a_{ \pm}^{*}(g), f, g \in L^{2}\left(\mathbb{R}^{\nu}\right)$, the annihilation and creation operators on $\mathscr{F}_{ \pm}$respectively. These operators satisfy the canonical commutation relations (CCRs)

$$
\left[a_{+}(f), a_{+}(g)\right]=0, \quad\left[a_{+}^{*}(f), a_{+}^{*}(g)\right]=0, \quad\left[a_{+}(f), a_{+}^{*}(g)\right]=(f, g) 1,
$$

and the canonical anticommutation relations (CARs)

$$
\left\{a_{-}(f), a_{-}(g)\right\}=0, \quad\left\{a_{-}^{*}(f), a_{-}^{*}(g)\right\}=0, \quad\left\{a_{-}(f), a_{-}^{*}(g)\right\}=(f, g) 1,
$$

where we used the notation $[A, B]=A B-B A$ and $\{A, B\}=A B+B A$. Notice that $\left\|a_{-}(f)\right\|=\left\|a_{-}^{*}(f)\right\|=\|f\|$. For details, see [3]. From now on, we suppress \pm in the notation if there is no confusion involved.

The CAR algebra is defined as follows: For each bounded region $\Lambda \subset \mathbb{R}^{\nu}$, define $\mathscr{A}_{\Lambda}$ as the von Neumann subalgebra generated by $\left\{a(f), a^{*}(g) \mid f, g \in L^{2}(\Lambda)\right\}$, where $a(f)$ and $a^{*}(f)$ satisfy the CARs in (3.2). Let $\mathscr{A}$ be the norm closure of $\bigcup_{\Lambda} \mathscr{b}_{\Lambda}$. It
then follows that $\left(\mathscr{A},\left\{\mathscr{b}_{\Lambda}\right\}\right)$ is a quasi-local algebra.

In order to describe the CCR algebra, let $\Phi(f)$ be the closure of $\left(a(f)+a^{*}(f)\right) / 2$, where $a(f)$ and $a^{*}(f)$ satisfy the CCRs in (3.1). Then for each $f \in L^{2}\left(\mathbb{R}^{\nu}\right), \Phi(f)$ is a self-adjoint operator on the symmetric Fock space $\mathscr{F}$. Let $W(f)$ denote the unitary operator $\exp \{i \Phi(f)\}, f \in L^{2}\left(\mathbb{R}^{\nu}\right)$. These operators satisfy the Weyl form of the CCRs:

$$
W(f) W(g)=\exp \{-i \operatorname{Im}(f, g) / 2\} W(f+g) .
$$

For each bounded region $\Lambda \subset \mathbb{R}^{\nu}$, define $\mathscr{A}_{\Lambda}$ as the von Neumann subalgebra generated by $\left\{W(f) \mid f \in L^{2}(\Lambda)\right\}$, and let $\mathscr{A}$ be the norm closure of $\bigcup_{\Lambda} \mathscr{A}_{\Lambda}$.
Then $\left(\mathscr{A},\left\{\mathscr{C}_{\Lambda}\right\}\right)$ is a quasi-local algebra.

Next we recall the gauge invariant quasi-free states on the CAR and CCR algebras [3]. If $0 \leq A \leq 1$ is an operator on $L^{2}\left(\mathbb{R}^{\nu}\right)$, then the quasi-free state on the CAR algebra is defined by

$$
\omega_{A}\left(a^{*}\left(f_{m}\right) \ldots a^{*}\left(f_{1}\right) a\left(g_{1}\right) \ldots a\left(g_{n}\right)\right)=\delta_{n m} \operatorname{det}\left(\left(g_{i}, A f_{j}\right)\right) .
$$

If $A$ is a bounded positive operator on $L^{2}\left(\mathbb{R}^{\nu}\right)$, the quasi-free state on the CCR algebra is defined by

$$
\omega_{A}(W(f))=\exp \left\{-\frac{1}{4}(f,(1+2 A) f)\right\} .
$$

A simple calculation shows that

$$
\omega_{A}\left(a_{ \pm}^{*}(f) a_{ \pm}(g)\right)=(g, A f),
$$

for any $f, g \in L^{2}\left(\mathbb{R}^{\nu}\right)$, and any higher order truncated functionals equal to zero [3]. 
In the rest of the paper we only consider the gauge invariant quasi-free states $\omega_{A}$ described above. For a given bounded positive operator $A(0 \leq A<1$ for the CAR algebra) on $L^{2}\left(\mathbb{R}^{\nu}\right)$, let $B$ be the operator defined by

$$
B=A /(1+\sigma A), \quad(\sigma= \pm 1) .
$$

Throughout this paper we shall use the convention that $\sigma=+1$ (resp. $\sigma=-1$ ) stands for the CCR (resp. CAR) algebra. Equivalently, for given bounded positive oeprator $B\left(0 \leq B<1\right.$ for the CCR algebra) on $L^{2}\left(\mathbb{R}^{\nu}\right)$, let $A$ be the operator defined by

$$
A=B /(1-\sigma B), \quad(\sigma= \pm 1)
$$

and let $\omega_{A}$ be the quasi-free state corresponding to $A$. It will be convenient to give conditions on $B$, instead of $A$. We assume that there exists an $L^{1}$ function $K_{B}: \mathbb{R}^{\nu} \rightarrow \mathbb{R}$ such that

$$
(B f)(x)=\int K_{B}(x-y) f(y) d^{\nu} y
$$

and let

$$
\hat{K}_{B}(k)=\int K_{B}(x) e^{-i k \cdot x} d^{\nu} x
$$

Similarly, let $K_{A}$ be the kernel function of $A$ :

$$
(A f)(x)=\int K_{A}(x-y) f(y) d^{\nu} y
$$

and $\hat{K}_{A}$ the Fourier transform of $K_{A}$ :

$$
\hat{K}_{A}(k)=\int K_{A}(x) e^{-i k \cdot x} d^{\nu} x
$$

Throughout this paper we assume that the following conditons hold:

Assumption 3.1. We assume that the following conditions are satisfied:

(a) There exist constants $0 \leq M_{1}<1$ and $0 \leq M_{2}$ such that $0 \leq \hat{K}_{B} \leq M_{1}$ for the CCR algebra and $0 \leq \hat{K}_{B} \leq M_{2}$ for the CAR algebra.

(b) There exist constants $0 \leq M_{3}$ and $0<\alpha$ such that the bound

holds.

$$
\left|\hat{K}_{B}(k)\right| \leq M_{3} \prod_{j=1}^{\nu}\left(1+\left|k_{\jmath}\right|\right)^{-(1+\alpha)}
$$

(c) Let $K_{B^{1 / 2}}(x)$ be the kernel function of the operator $B^{1 / 2}$, i.e., the inverse Fourier transform of $\left(\hat{K}_{B}\right)^{1 / 2}$. There exist constants $0 \leq M_{4}$ and $0<\alpha$ such that the bound

holds.

$$
\left|K_{B^{1 / 2}}(x)\right| \leq M_{4} \prod_{\jmath=1}^{\nu}\left(1+\left|x_{j}\right|\right)^{-(1+\alpha)}
$$

Remark 3.2. (a) Assumption 3.1(a) implies that $0 \leq B \leq M_{1}<1$ for the CCR algebra and $0 \leq B \leq M_{2}$ for the CAR algebra.

(b) Since

$$
\hat{K}_{A}=\hat{K}_{B} /\left(1-\sigma \hat{K}_{B}\right), \quad(\sigma= \pm 1) .
$$

Assumption 3.1(a) and (b) imply $\hat{K}_{A}$ is integrable. 
(c) We impose Assumption 3.1(c) to obtain a key technical estimate (the last inequality in Lemma 5.2). If $D_{k_{1}}^{\beta_{1}} \ldots D_{k_{\nu}}^{\beta_{\nu}}\left(\hat{K}_{B}\right)^{1 / 2} \in L^{1}$ for any $\beta=\left(\beta_{1}, \ldots, \beta_{\nu}\right)$ with $\beta_{\imath} \leq 3, i=1, \ldots, \nu$, then the assumption holds. Since the condition is imposed on $K_{B^{1 / 2}}$ instead of $K_{B}$, it would be nice if one can replace it by a condition on $K_{B}$. (d) Since $K_{B}(x)=\left(K_{B^{1 / 2}} * K_{B^{1 / 2}}\right)(x)$, it follows from Assumption 3.1(c) that there is a constant $M_{5}$ such that the bound

holds.

$$
\left|K_{B}(x)\right| \leq M_{5} \prod_{\jmath=1}^{\nu}\left(1+\left|x_{j}\right|\right)^{-(1+\alpha)}
$$

(e) The states for ideal Fermi and Bose gases at inverse temperature $\beta=1 / T$ with activity $z=e^{\mu}$ are the quasi-free states corresponding to

$$
\hat{K}_{B}(k)=\exp \left(-\beta k^{2}+\mu\right) \quad(\mu<0 \text { for the CCRs }) .
$$

Obviously all conditions in Assumption 3.1 are satisfied in these cases.

We consider the unit step space translations ( $\mathbb{Z}^{\nu}$-action) on the CAR and CCR algebras. We will use the following notation:

$$
\begin{gathered}
\vec{n}=\left(n_{1}, n_{2}, \ldots, n_{\nu}\right) \in \mathbb{Z}^{\nu}, \\
B(\vec{k})=B\left(k_{1}, \ldots, k_{\nu}\right)=\left\{\vec{n} \in \mathbb{Z}^{\nu} \mid 0 \leq n_{\text {J }}<k_{j}, j=1, \ldots, \nu\right\} .
\end{gathered}
$$

For each $\vec{n} \in \mathbb{Z}^{\nu}$, let $U(\vec{n})$ be the unitary operator on $L^{2}\left(\mathbb{R}^{\nu}\right)$ defined by

$$
(U(\vec{n}) f)(x)=f(x-\vec{n}) .
$$

Then $U(\vec{n})$ induces an automorphism $\theta^{\vec{n}}$ on the CAR and CCR algebras by

$$
\theta^{\vec{n}}\left(a^{*}(f)\right)=a^{*}(U(\vec{n}) f) .
$$

Obviously the quasi-free states $\omega_{A}$ are invariant under $\theta^{\vec{n}}, \vec{n} \in \mathbb{Z}^{\nu}$. For a completely positive unital map $\gamma: \mathscr{N}^{\cdot} \rightarrow \mathscr{A}$ from a finite dimensional $C^{*}$-algebra $\mathscr{N}^{\cdot}$ to $\mathscr{A}$, and for the $\mathbb{Z}^{\nu}$-action $\theta: A, \rightarrow A$, denote by

$$
h_{\omega_{A}, \theta}(\gamma)=\lim _{\substack{k_{\jmath} \rightarrow \infty \\ \jmath=1, \ldots, \nu}} \frac{1}{k_{1} \ldots k_{\nu}} H\left(\left(\theta^{\vec{n}} \circ \gamma\right): \vec{n} \in B(\vec{k})\right) .
$$

The above limit exists by the subadditivity of the entropy functional $H_{\omega_{A}}\left(\gamma_{1}, \ldots, \gamma_{n}\right)$. The dynamical entropy of the space translations with respect to the quasi-free states $\omega_{A}$ on the CAR and CCR algebras is defined by

$$
h_{\omega_{A}}(\theta)=\sup _{\gamma} h_{\omega_{A}, \theta}(\gamma) \text {. }
$$

It may be worth to compare the above definition to that in (2.7) and (2.8).

For any bounded region $\Lambda \subset \mathbb{R}^{\nu}$, let $\chi_{\Lambda}$ denote the projection operator from $L^{2}\left(\mathbb{R}^{\nu}\right)$ onto $L^{2}(\Lambda)$, and let

$$
A_{\Lambda}=\chi_{\Lambda} A \chi_{\Lambda}
$$

and let $|\Lambda|$ be the volume of $\Lambda$. From (3.11),(3.12), Assumption 3.1(b) and Remark 3.2 (b), it follows that

$$
\operatorname{Tr}_{L^{2}(\Lambda)}\left(A_{\Lambda}\right)=(2 \pi)^{-\nu}|\Lambda| \int \hat{K}_{A}(k) d^{\nu} k<\infty .
$$


and so the state $\omega_{A}$ is a locally normal [3]. Let $\varrho_{A}$ be the density operator on $\mathscr{F}_{\Lambda}$ corresponding to $\omega_{\Lambda}=\left.\omega_{A}\right|_{\mathscr{B}_{\Lambda}}$. The local entropy for the state $\omega_{\Lambda}$ is defined by

$$
S\left(\omega_{\Lambda}\right) \equiv-\operatorname{Tr}_{\mathscr{F}_{\Lambda}}\left(\varrho_{\Lambda} \log \varrho_{\Lambda}\right)
$$

and the mean entropy is defined by

$$
s\left(\omega_{A}\right) \equiv \lim _{\Lambda \uparrow \mathbb{R}^{\nu}} \frac{1}{|\Lambda|} S\left(\omega_{\Lambda}\right)
$$

The above limit exists by the subadditivity of $S\left(\omega_{\Lambda}\right)$ [3]. We now state our main results:

Theorem 3.3. Let $\left(\mathscr{A},\left\{\mathscr{A}_{\Lambda}\right\}\right)$ denote the CAR and CCR quasi-local algebras, $\theta$ the space translation automorphism on $\mathscr{C}$ and $\omega_{A}$ the quasi-free state on $\mathscr{A}$. Under the assumptions in Assumption 3.1, the dynamical entropies $h_{\omega_{A}}(\theta)$ for the CAR and CCR algebras are equal to the corresponding mean entropies $s\left(\omega_{A}\right)$.

Theorem 3.4. Let $s\left(\omega_{A}\right)$ be the mean entropy for the quasi-free state $\omega_{A}$. Under Assumption 3.1 the following results hold:

(a) For the CAR algebra the formula

$$
s\left(\omega_{A}\right)=-(2 \pi)^{-\nu} \int\left\{\hat{K}_{A}(k) \log \hat{K}_{A}(k)+\left(1-\hat{K}_{A}(k)\right) \log \left(1-\hat{K}_{A}(k)\right)\right\} d^{\nu} k
$$

holds.

(b) For the CCR algebra the formula

$$
s\left(\omega_{A}\right)=-(2 \pi)^{-\nu} \int\left\{\hat{K}_{A}(k) \log \hat{K}_{A}(k)-\left(1+\hat{K}_{A}(k)\right) \log \left(1+\hat{K}_{A}(k)\right)\right\} d^{\nu} k
$$

holds.

The main part of the paper will be devoted to proof of Theorem 3.3. The proof of Theorem 3.4 will be given in Appendix.

\section{Reduction of the Proof of Theorem 3.3}

In this section we reduce the proof of Theorem 3.3 to the vanishing of the mean entropy defect (Proposition 4.3). We first derive a upper bound for the dynamical entropy and then state Proposition 4.3 which implies that the average entropy defects tend to zero as $\Lambda$ tends to $\mathbb{R}^{\nu}$. Using Proposition 4.3 we derive a lower bound for the dynamical entropy, which completes the proof of Theorem 3.3. The proof of Proposition 4.3 will be postponed to the following sections. The following is the upper bound for the dynamical entropy.

Proposition 4.1. Let $h_{\omega_{A}}(\theta)$ and $s\left(\omega_{A}\right)$ be the dynamical and mean entropies defined in (3.17) and (3.21) respectively. For both CAR and CCR algebras, the bound

holds.

$$
h_{\omega_{A}}(\theta) \leq s\left(\omega_{A}\right)
$$

Proof. As a consequence of Theorem 2.2 for the CCR algebra and Theorem 2.4 for the CAR algebra, it follows that

$$
h_{\omega_{A}}(\theta)=\lim _{\Lambda \uparrow \mathbb{R}^{\nu}} \lim _{n \rightarrow \infty} h_{\omega_{A}, \theta}\left(\mathscr{A}_{\Lambda, n}\right),
$$


where

$$
h_{\omega_{A}, \theta}\left(\mathscr{C}_{\Lambda, n}\right)=\lim _{\substack{k_{j} \rightarrow \infty \\ \jmath=1, \ldots, \nu}} \frac{1}{k_{1} \ldots k_{\nu}} H\left(\left(\theta^{\vec{n}}\left(\mathscr{C}_{\Lambda, n}\right): \vec{n} \in B(\vec{k})\right) .\right.
$$

We assert that the algebra $\mathscr{C}(\Lambda ; B(\vec{k}))$ generated by $\left\{\theta^{\vec{n}}\left(\mathscr{C}_{\Lambda, n}\right): \vec{n} \in B(\vec{k}\}\right.$ is finite dimensional. Let $\mathscr{A}(\Lambda ;\{\vec{m}\})$ be the algebra generated by $\left\{\left(\mathscr{C}_{\Lambda, n}\right), \theta^{\vec{m}}\left(\mathscr{C}_{\Lambda, n}\right)\right\}$. We first show that $\mathscr{b}(\Lambda ;\{\vec{m}\}), \vec{m} \in \mathbb{Z}^{\nu}$, is finite dimensional. Denote by $\mathscr{F}_{\Lambda, n}$ the finite dimensional subspace of $\mathscr{F}_{\Lambda}$ spanned by eigenvectors $\left\{\phi_{1}, \ldots, \phi_{n}\right\}$ of $\varrho_{\Lambda}$, and denote by $P_{\Lambda, n}$ the projection operator from $\mathscr{F}_{\Lambda}$ (and also from $\mathscr{F}$ ) to $\mathscr{F}_{\Lambda, n}$. Recall from (2.9) that

$$
\mathscr{C}_{\Lambda, n}=P_{\Lambda, n} \mathscr{C}_{\Lambda} P_{\Lambda, n} \oplus \mathbb{C} \cdot P_{\Lambda, n}^{\perp} .
$$

Obviously $\mathscr{C}_{\Lambda, n}$ is generated by the finite dimensional algebra $P_{\Lambda, n} \mathscr{b}_{\Lambda} P_{\Lambda, n}$ and the identity 1 . Let $\mathscr{F}(\Lambda ;\{\vec{m}\})$ be the finite dimensional subspace of $\mathscr{F}$ spanned by the vectors in either $\mathscr{F}_{\Lambda, n}$ or $\theta^{\vec{m}}\left(P_{\Lambda, n}\right) \mathscr{F}$. Then the algebra $\mathscr{B}(\Lambda ;\{\vec{m}\})$ generated by $P_{\Lambda, n} \mathscr{A}_{\Lambda} P_{\Lambda, n}$ and $\theta^{\vec{m}}\left(P_{\Lambda, n} \mathscr{C}_{\Lambda} P_{\Lambda, n}\right)$ is finite dimensional represented $C^{*}$-algebra on $\mathscr{F}(\Lambda ;\{\vec{m}\})$. Since $\mathscr{B}(\Lambda ;\{\vec{m}\})$ is also generated by $\mathscr{B}(\Lambda ;\{\vec{m}\})$ and the identity 1 , it is finite dimensional. The argument employed above shows that $\mathscr{A}(\Lambda ; B(\vec{k}))$ is finite dimensional. This proved our assertion.

By the monotonicity of the entropy functionals [7, Proposition III.6(a)] and by [7, Proposition III.6(b)] it follows that

$$
\begin{aligned}
H\left(\left(\theta^{\vec{n}}\left(\mathscr{C}_{\Lambda, n}\right)\right): \vec{n} \in B(\vec{k})\right) & \leq H(\mathscr{C}(\Lambda ; B(\vec{k})), \ldots, \mathscr{C}(\Lambda ; B(\vec{k}))) \\
& =H(\mathscr{C}(\Lambda ; B(\vec{k}))) .
\end{aligned}
$$

We choose $\Lambda$ by $\Lambda=(-L / 2, L / 2)^{\nu}$. Denote by $\Lambda(\vec{k})$ the set $\{x+\vec{n} \mid x \in \Lambda, \vec{n} \in$ $B(\vec{k})\}$. As a consequence of Proposition 4.4 of [16], it follows that

$$
\begin{aligned}
H(\mathscr{A}(\Lambda ; B(\vec{k}))) & \leq \lim _{n \rightarrow \infty} H\left(\mathscr{C}_{\Lambda(\vec{k}), n}\right) \\
& \leq \lim _{n \rightarrow \infty} S\left(\mathscr{C}_{\Lambda(\vec{k}), n}\right)
\end{aligned}
$$

where $S\left(\mathscr{A}_{\Lambda(\vec{k}), n}\right)$ is the entropy for the state $\left.\omega_{A}\right|_{\ell_{\Lambda(\vec{k}), n}}$. To get the second inequality we have used the fact that $H\left(\mathscr{A}_{\Lambda, n}\right) \leq S\left(\mathscr{A}_{\Lambda, n}\right)$ for any $\Lambda$.

Let $\left\{\lambda_{i}\right\}$ be the eigenvalues of $\varrho_{\Lambda(\vec{k})}$ listed in the decreasing order. A direct computation shows that

$$
\begin{aligned}
\lim _{n \rightarrow \infty} S\left(\mathscr{C}_{\Lambda(k), n}\right) & =-\lim _{n \rightarrow \infty}\left\{\sum_{\imath=1}^{n} \lambda_{i} \log \lambda_{i}+\left(\sum_{j=n+1}^{\infty} \lambda_{j}\right) \log \left(\sum_{j=n+1}^{\infty} \lambda_{j}\right)\right\} \\
& =-\sum_{i=1}^{\infty} \lambda_{\imath} \log \lambda_{i}=S\left(\omega_{\Lambda(\vec{k})}\right)
\end{aligned}
$$

where $S\left(\omega_{\Lambda(\vec{k})}\right)$ is the local entropy defined in (3.20). Thus from (4.1)-(4.4) it follows that

$$
h_{\omega_{A}, \theta}\left(\mathscr{C}_{\Lambda, n}\right) \leq \lim _{\substack{k_{j} \rightarrow \infty \\ j=1, \ldots, \nu}} \frac{1}{k_{1} \ldots k_{\nu}} S\left(\omega_{\Lambda(\vec{k})}\right)=\lim _{\substack{k_{j} \rightarrow \infty \\ j=1, \ldots, \nu}} \frac{1}{|\Lambda(\vec{k})|} S\left(\omega_{\Lambda(\vec{k})}\right)=s\left(\omega_{A}\right),
$$


where $s\left(\omega_{A}\right)$ is the mean entropy defined in (3.21). This proved the proposition completely. Q.E.D.

We consider the entropy functional $H$ defined in Sect. 2 in more details. For multiindex $I=\left(i_{1}, \ldots, i_{n}\right)$, let $\left\{\omega_{I}\right\}_{I}$ be a decomposition of the state $\omega$ :

$$
\omega_{I}=\omega_{i_{1}, \ldots, i_{n}}, \quad \sum_{I} \omega_{I}=\omega .
$$

Denote that $\eta(x)=-x \log x$. Let

$$
\omega_{i_{k}}^{(k)} \equiv \sum_{\substack{i_{1}, \ldots, i_{n} \\ i_{k}: \text { fixed }}} \omega_{i_{1}, \ldots, i_{n}}, \quad \hat{\omega}_{i_{k}}^{(k)}=\omega_{i_{k}}^{(k)} / \omega_{i_{k}}^{(k)}(1) .
$$

Let $\mathscr{A}_{\imath}, i=1, \ldots, n$, be finite dimensional subalgebras of $\mathscr{A}_{\text {. Then the entropy }}$ function can be written as

$$
\begin{aligned}
H_{\omega}\left(\mathscr{b}_{1}, \ldots, \mathscr{A}_{n}\right)= & \sup _{\sum_{I} \omega_{I}=\omega}\left[\sum_{I} \eta\left(\omega_{I}(1)\right)-\sum_{k=1}^{n} \sum_{i_{k}} \eta\left(\omega_{i_{k}}^{(k)}(1)\right)\right. \\
& \left.+\sum_{k=1}^{n} S\left(\left.\omega\right|_{\ell_{k}}\right)-\sum_{k=1}^{n} \sum_{i_{k}} \omega_{i_{k}}^{(k)}(1) S\left(\left.\hat{\omega}_{i_{k}}^{(k)}\right|_{\iota_{k}}\right)\right] .
\end{aligned}
$$

For details, see [7].

In order to get a lower bound for the entropy functional, one has to choose a decomposition of the state, $\omega=\sum \omega_{I}$, as much as close as possible to the optimal decomposition. In general a decomposition can be written

$$
\omega_{I}(a)=\omega\left(x_{I}^{\prime} a\right)=\omega\left(\sigma_{i / 2}\left(x_{I}\right) a\right)
$$

with $x_{I}^{\prime} \in \pi_{\omega}(\mathscr{A})^{\prime}, x_{I} \in \pi_{\omega}(\mathscr{A})^{\prime \prime}, \sigma_{t}$ the modular automorphism of $\omega[1,3]$. In our case, we shall choose $x_{I}$ to be the projection operator $P_{I}$ consisting of the spectral projections of the local density operator $\varrho_{\Lambda}$.

To be more precise, let $B^{\Lambda}$ be the operator on $L^{2}(\Lambda)$ defined by

$$
B^{\Lambda}=A_{\Lambda} /\left(1+\sigma A_{\Lambda}\right), \quad(\sigma= \pm 1),
$$

where $A_{\Lambda}=\chi_{\Lambda} A \chi_{\Lambda}$. Let $B$ the operator related to $A$ by (3.7). Let $h$ and $h_{\Lambda}$ be the self-adjoint operators defined by

$$
\begin{array}{cc}
\sigma_{t}\left(a^{*}(f)\right)=a^{*}\left(e^{-i t h} f\right), & f \in L^{2}\left(\mathbb{R}^{\nu}\right), \\
\sigma_{t}^{\Lambda}\left(a^{*}(g)\right)=a^{*}\left(e^{-i t h} \Lambda\right), & g \in L^{2}(\Lambda)
\end{array}
$$

respectively, where $\sigma_{t}^{\Lambda}$ be the modular automorphism of $\omega_{\Lambda}=\left.\omega\right|_{\ell_{\Lambda}}$. From the KMS conditions $\omega\left(x \sigma_{-i}(y)\right)=\omega(y x)$ and $\omega_{\Lambda}\left(x^{\prime} \sigma_{-\imath}^{\Lambda}\left(y^{\prime}\right)\right)=\omega_{\Lambda}\left(y^{\prime} x^{\prime}\right)$, it follows that

$$
\begin{gathered}
\omega\left(a(g) a^{*}\left(e^{-h} f\right)\right)=\omega\left(a^{*}(f) a(g)\right), \quad f, g \in L^{2}\left(\mathbb{R}^{\nu}\right), \\
\omega_{\Lambda}\left(a\left(g^{\prime}\right) a^{*}\left(e^{-h_{\Lambda}} f^{\prime}\right)\right)=\omega_{\Lambda}\left(a^{*}\left(f^{\prime}\right) a\left(g^{\prime}\right)\right), \quad f^{\prime}, g^{\prime} \in L^{2}(\Lambda),
\end{gathered}
$$

and so by (3.1), (3.2), and (3.6)

$$
\begin{aligned}
\left(g,(1+\sigma A) e^{-h} f\right) & =(g, A f), \quad f, g \in L^{2}\left(\mathbb{R}^{\nu}\right), \\
\left(g^{\prime},\left(1+\sigma A_{\Lambda}\right) e^{-h_{\Lambda}} f^{\prime}\right) & =\left(g^{\prime}, A_{\Lambda} f^{\prime}\right), \quad f^{\prime}, g^{\prime} \in L^{2}(\Lambda),
\end{aligned}
$$


respectively. From (3.7), (4.6) and the above relations, we conclude that the relations

$$
B=\exp (-h), \quad B^{\Lambda}=\exp \left(-h_{\Lambda}\right)
$$

hold. Thus the operators $B$ and $B^{\Lambda}$ are closely related to the modular automorphisms $\sigma_{t}$ and $\sigma_{t}^{\Lambda}$ respectively. The argument used above can also be found in [22].

Now we are in the position to give a decomposition of the state $\omega_{A}$. By Assumption 3.1(b), it can be checked that the operator $B^{\Lambda}$ defined in (4.6) is of trace class. See Lemma 5.2 in the next section. Let $\left\{\gamma_{k}\right\}_{k=1}^{\infty}$ be the eigenvalues of $h_{\Lambda}$ listed in increasing order and counting the multiplicities, and let $\left\{f_{k}\right\}_{k=1}^{\infty}$ be the corresponding normalized eigenvectors. We describe the family of projection operators $\left\{P_{I}\right\}_{I}$, which will give a decomposition of $\omega_{A}$, separately for the CAR algebra and for the CCR algebra.

The case of the CAR algebra: Denote for $k \in \mathbb{N}$

$$
N\left(f_{k}\right)=a^{*}\left(f_{k}\right) a\left(f_{k}\right), \quad \hat{N}\left(f_{k}\right)=1-N\left(f_{k}\right)=a\left(f_{k}\right) a^{*}\left(f_{k}\right),
$$

and

$$
P_{k}(0)=\hat{N}\left(f_{k}\right), \quad P_{k}(1)=N\left(f_{k}\right) .
$$

By the CARs, $P_{k}(0)$ and $P_{k}(1)$ are projection operators with $P_{k}(0)+P_{k}(1)=1$. For given (fixed) $N \in \mathbb{N}$, let

$$
I=\left(i_{1}, i_{2}, \ldots, i_{N}\right) \in\{0,1\}^{N}
$$

be a multi-index, and let

$$
P_{I}=P_{1}\left(i_{1}\right) P_{2}\left(i_{2}\right) \ldots P_{N}\left(i_{N}\right)
$$

Then $\left\{P_{I}: I \in\{0,1\}^{N}\right\}$ is a family of mutually commuting orthogonal projections satisfying

$$
\sum_{I} P_{I}=1
$$

Denote by $\mathscr{A}_{\Lambda}^{(N)}$ the finite dimensional abelian $C^{*}$-algebra generated by $\left\{P_{I}: I \in\right.$ $\left.\{0,1\}^{N}\right\}$. Clearly one has that $\mathscr{A}_{\Lambda}^{(N)} \subset \mathscr{A}_{\Lambda}$.

The case of the CCR algebra: As in (4.9), let $N\left(f_{\imath}\right)$ be the number operator for the state $f_{i}, N\left(f_{\imath}\right)=a^{*}\left(f_{i}\right) a\left(f_{i}\right)$. Then for each $i \in \mathbb{N}, N\left(f_{\imath}\right)$ has pure point spectrum $\mathbb{Z}_{+}$, where $\mathbb{Z}_{+}=\mathbb{N} \cup\{0\}$. Let $\left\{Q_{i}(n): n \in \mathbb{Z}_{+}\right\}$be the family of spectral projections of $N\left(f_{i}\right)$. Denote for given (fixed) $N \in \mathbb{N}$,

$$
\begin{gathered}
P_{\imath}(k)=Q_{i}(k), \quad 0 \leq k<N, i \in \mathbb{N}, \\
P_{i}(N)=1-\sum_{k=0}^{N-1} Q_{\imath}(k) .
\end{gathered}
$$

For given (fixed) $N \in \mathbb{N}$, let

$$
I=\left(i_{1}, \ldots, i_{N}\right) \in\{0,1, \ldots, N\}^{N}
$$

be a multi-index, and let

$$
P_{I}=P_{1}\left(i_{1}\right) P_{2}\left(i_{2}\right) \ldots P_{N}\left(i_{N}\right) .
$$


Then $\left\{P_{I}: I \in\{0,1, \ldots, N\}^{N}\right\}$ be a family of mutually commuting orthogonal projections satisfying $\sum_{I} P_{I}=1$. Denote by $\mathscr{A}_{\Lambda}^{(N)}$ the finite dimensional abelian $C^{*}$-algebra generated by $\left\{P_{I}\right\}_{I}$.

We come back to the entropy functional in (4.5). From now on, we choose $\Lambda$ as $\Lambda=(0, L)^{\nu}$, where $L$ is a positive integer. Let $B(\vec{k})$ be defined as in (3.14) and let

$$
B(L \vec{k})=\left\{\vec{n}=\left(n_{1}, \ldots, n_{\nu}\right) \in \mathbb{Z}^{\nu}: 0 \leq n_{j}<L k_{\jmath}, j=1, \ldots, \nu\right\} .
$$

Let $\mathscr{C}_{\Lambda}^{(N)}$ be the abelian $C^{*}$-algebra generated by $\left\{P_{I}\right\}_{I}$ defined as above. Then from [7, Proposition III.6.d] it follows that

$$
H_{\omega}\left(\left(\theta^{\vec{n}}\left(\mathscr{t}_{\Lambda}^{(N)}\right)\right): \vec{n} \in B(L \vec{k})\right) \geq H_{\omega}\left(\left(\theta^{L \vec{n}}\left(\mathscr{A}_{\Lambda}^{(N)}\right)\right): \vec{n} \in B(\vec{k})\right) .
$$

From (3.16), (3.17) and the above inequality, we conclude that the bound

$$
h_{\omega}(\theta) \geq \lim _{L \rightarrow \infty} \lim _{\substack{k_{\jmath} \rightarrow \infty \\ \jmath=1, \ldots, \nu}} \frac{1}{\left(L k_{1}\right) \ldots\left(L k_{\nu}\right)} H_{\omega}\left(\left(\theta^{L \vec{n}}\left(A_{\Lambda}^{(N)}\right)\right): \vec{n} \in B(\vec{k})\right)
$$

holds. We choose the decomposition of the state $\omega, \sum_{J} \omega_{J}=\omega$, as follows: Let $J=\left(I_{\vec{n}}: \vec{n} \in B(\vec{k})\right)$ be a multi-index, where for each $\vec{n} \in B(\vec{k}), I_{\vec{n}} \in\{0,1\}^{N}$ for the CARs and $I_{\vec{n}} \in\{0,1, \ldots, N\}^{N}$ for the CCRs. Let

$$
\omega_{J}(a)=\omega\left(\sigma_{i / 2}\left(x_{J}\right) a\right),
$$

where for $J=\left(I_{\vec{n}}: \vec{n} \in B(\vec{k})\right)$

$$
x_{J}=\prod_{\vec{n} \in B(\vec{k})}\left(\theta^{L \vec{n}}\left(P_{I_{\vec{n}}}\right)\right) .
$$

Notice that $\sum_{J} x_{J}=1$, and so (4.18) is a decomposition of the state $\omega$.

Remark 4.2. (a) A comment on the decomposition of the state $\omega$ given above is in order. Since $\sigma_{i / 2}(N(f))$ is not defined as an element of. $\mathscr{b}$ if $f$ is not in $D\left(B^{-1 / 2}\right)$, one may think that there is a domain problem in the decomposition. But there is no domain problem by the following reason. Let $\left(\mathscr{H}_{\omega}, \pi_{\omega}(\mathscr{\ell}), \Omega_{\omega}\right)$ be the cyclic representation of $\mathscr{h}$, and let $\Delta$ and $J$ be the modular operator and the modular conjugation associated with $\left\{\pi_{\omega}(\mathscr{b})^{\prime \prime}, \Omega_{\omega}\right\}$ respectively [3, Sect. 2.5.2]. By $\omega\left(\sigma_{i / 2}(x) y\right)$ we really mean that for any $x, y \in \mathscr{A}$,

$$
\omega\left(\sigma_{i / 2}(x) y\right) \equiv\left(\Omega_{\omega} ; \pi_{\omega}(x) \Delta^{1 / 2} \pi_{\omega}(y) \Omega_{\omega}\right)
$$

Since $\pi_{\omega}(\mathscr{b})^{\prime \prime} \Omega_{\omega} \subset D\left(\Delta^{1 / 2}\right)$, the r.h.s. of the above is well-defined. Throughout this paper we use the above notation. Notice that the r.h.s. of the above equals $\left(\Omega_{\omega}, \pi_{\omega}(x) J \pi_{\omega}(y) J \Omega_{\omega}\right)$ and so (4.18) defines a positive linear functional on $\mathscr{A}_{\text {. }}$.

(b) Using the above notation, (4.7) and the modular condition [3], it can be checked that for any $y \in \mathscr{A}$ and $f \in L^{2}\left(\mathbb{R}^{\nu}\right)$,

$$
\omega\left(\sigma_{\imath / 2}\left(a^{*}(f) a(f)\right) y\right)=\omega\left(\left(a\left(B^{1 / 2} f\right) y a^{*}\left(B^{1 / 2} f\right)\right) .\right.
$$

This is one version of the KMS conditon which will be used frequently in the sequel. Even if $N(f)$ is a unbounded operator for each $f \in L^{2}\left(\mathbb{R}^{\nu}\right)$ in the case of the CCR 
algebra, the above relation make sense by the fact that quasi-free states are regular [3].

Using the decomposition in (4.18) and (4.19), we obtain from (4.5) that

$$
\begin{aligned}
H\left(\left(\theta^{L \vec{n}}\left(\mathscr{C}_{\Lambda}^{(N)}\right)\right): \vec{n} \in B(\vec{k})\right) \geq & \sum_{J=\left(I_{\vec{n}}: \vec{n} \in B(\vec{k})\right)} \eta\left(\omega\left(\prod_{\vec{n} \in B(\vec{k})}\left(\theta^{L \vec{n}}\left(P_{I_{\vec{n}}}\right)\right)\right)\right) \\
& -\left(k_{1} \ldots k_{\nu}\right) \sum_{I} \omega\left(P_{I}\right) S\left(\left.\hat{\omega}_{I}\right|_{\left.\mathcal{H}_{\Lambda}^{(N)}\right)}\right.
\end{aligned}
$$

where $\hat{\omega}_{I}(a)=\omega\left(\sigma_{i / 2}\left(P_{I}\right) a\right) / \omega\left(P_{I}\right)$. Here we have used the fact that

$$
S\left(\left.\omega\right|_{\mathscr{R}_{\Lambda}^{(N)}}\right)=\sum \eta\left(\omega\left(P_{I}\right)\right)
$$

which follows from the definitions of $P_{I}$ and $\mathscr{C}_{\Lambda}^{(N)}$ in (4.12) (resp. (4.16)) and the below of (4.13) (resp. (4.16)). To simplify the notation, let us write

$$
\begin{gathered}
E(\Lambda, N, \vec{k}) \equiv \sum_{J=\left(I_{\vec{n}}: \vec{n} \in B(\vec{k})\right)} \eta\left(\omega\left(\prod_{\vec{n} \in B(\vec{k})}\left(\theta^{L \vec{n}}\left(P_{I_{\vec{n}}}\right)\right)\right)\right) \\
D(\Lambda, N) \equiv \sum_{I} \omega\left(P_{I}\right) S\left(\left.\hat{\omega}_{I}\right|_{\mathscr{C}_{\Lambda}^{(N)}}\right) .
\end{gathered}
$$

Then the inequality (4.20) becomes

$$
H\left(\left(\theta^{L \vec{n}}\left(\mathscr{C}_{\Lambda}^{(N)}\right)\right): \vec{n} \in B(\vec{k})\right) \geq E(\Lambda, N, \vec{k})-\left(k_{1} \ldots k_{\nu}\right) D(\Lambda, N) .
$$

The quantity $E(\Lambda, N, \vec{k})$ is the entropy of the state $\omega$ on the finite dimensional abelian $C^{*}$-algebra generated by $\left.\left\{\theta^{L \vec{n}}\left(\mathscr{C}_{\Lambda}^{(N)}\right)\right): \vec{n} \in B(\vec{k})\right\}$, and $D(\Lambda, N)$ is the entropy defect of the decomposition (4.18) of the state $\omega$ on $\mathscr{A}_{\Lambda}^{(N)}$.

Proposition 4.3. Let $D(\Lambda, N)$ be the entropy defect defined as in (4.21). For both CAR and CCR algebras, there exists a constant $c$ independent of $\Lambda$ and $N$ such that the bound

holds.

$$
L^{-\nu}|D(\Lambda, N)| \leq c L^{-1 / 8}
$$

Recall that $\Lambda=(0, L)^{\nu}$. Thus the above proposition implies that the average of the entropy defects tends to zero as $L \rightarrow \infty$. We postpone the proof of Proposition 4.3 to Sect. 6.

In the remainder of this section we shall prove our main result, Theorem 3,3 by using the above proposition and Proposition 4.1. First we have the following result:

Proposition 4.4. Let $E(\Lambda, N, \vec{k})$ be the quantity defined in (4.21). For given $\Lambda=$ $(0, L)^{\nu} \subset \mathbb{R}^{\nu}$ and $\varepsilon>0$, there exists $N_{0} \in \mathbb{N}$ such that for $N \geq N_{0}$ the bound

$$
S\left(\omega_{\Lambda(L \vec{k})}\right) \leq E(\Lambda, N, \vec{k})+\left(k_{1} \ldots k_{\nu}\right) \varepsilon
$$

holds for both CAR and CCR algebras, where $\Lambda(L \vec{k})=\left\{x: 0 \leq x_{j}<L k_{\jmath}, j=\right.$ $1, \ldots, \nu\}$.

Proof of Theorem 3.3. From (4.17), (4.22), Proposition 4.3 and Proposition 4.4 it follows that

$$
h_{\omega}(\theta) \geq|\Lambda(L \vec{k})|^{-1} S\left(\omega_{\Lambda(L \vec{k})}\right)-\varepsilon-c L^{-1 / 8},
$$


where $\omega_{\Lambda(L \vec{k})}=\left.\omega\right|_{\Lambda(L \vec{k})}$. We choose $L$ sufficiently large so that $c L^{-1 / 8}<\varepsilon$ and

$$
\left.|| \Lambda(L \vec{k})\right|^{-1} S\left(\omega_{\Lambda(L \vec{k})}\right)-s(\omega) \mid<\varepsilon .
$$

The above inequalities imply that for any $\varepsilon>0$,

$$
h_{\omega}(\theta) \geq s(\omega)-3 \varepsilon
$$

Now Theorem 3.3 follows from the above bound and Proposition 4.1. This proved Theorem 3.3 completely. Q.E.D.

In the rest of this section we prove Proposition 4.4.

Proof of Proposition 4.4.(a) The case of the CAR algebra: For given $N \in \mathbb{N}$, denote that

$$
I(\leq N)=\{1,2, \ldots, N\}, \quad I(>N)=\{n \in \mathbb{N}: n>N\}
$$

For any finite $J \subset I(>N)$, let

$$
P_{J}=\prod_{\jmath \in J} N\left(f_{\jmath}\right) \prod_{k \in J^{c}}\left(1-N\left(f_{k}\right)\right)
$$

Notice that

$$
\sum_{I \in\{0,1\}^{N}} \sum_{J \subset I(>N)} P_{I} P_{J}=1
$$

We define a density operator on $\mathscr{F}_{\Lambda(L \vec{k})}$ by

$$
\begin{aligned}
& \hat{\varrho}_{\Lambda(L \vec{k})}=\sum_{\substack{I_{\vec{n}} \in\{0,1\}^{N} \\
\vec{n} \in B(\vec{k})}} \sum_{\substack{J_{\vec{n}} \subset I(>N) \\
\vec{n} \in B(\vec{k})}} \omega\left(\prod_{\vec{n} \in B(\vec{k})}\left(\theta^{L \vec{n}}\left(P_{I_{\vec{n}}} P_{J_{\vec{n}}}\right)\right)\right) \\
& \times \prod_{\vec{n} \in B(\vec{k})} \theta^{L \vec{n}}\left(P_{I_{\vec{n}}} P_{J_{\vec{n}}}\right) .
\end{aligned}
$$

We use the inequality

$$
-\operatorname{Tr}(A(\log A-\log B)) \leq \operatorname{Tr}(B-A)
$$

for any positive operators $A$ and $B$ [3] and the fact that $\operatorname{Tr}\left(\varrho_{\Lambda(L \vec{k})} x\right)=\omega(x)$ for any $x \in \mathscr{A}_{\Lambda(L \vec{k})}$ to obtain that

$$
\begin{aligned}
& S\left(\left.\omega\right|_{\Lambda(L \vec{k})}\right)=-\operatorname{Tr}\left(\varrho_{\Lambda(L \vec{k})} \log \varrho_{\Lambda(L \vec{k})}\right) \\
& \leq-\operatorname{Tr}\left(\varrho_{\Lambda(L \vec{k})} \log \hat{\varrho}_{\Lambda(L \vec{k})}\right) \\
& =\sum_{I_{\vec{n}} \in\{0,1\}^{N}} \sum_{J_{\vec{n}} \subset I(>N)} \eta\left(\omega\left(\prod_{\vec{n} \in B(\vec{k})}\left(\theta^{L \vec{n}}\left(P_{I_{\vec{n}}} P_{J_{\vec{n}}}\right)\right)\right)\right) . \\
& \vec{n} \in B(\vec{k}) \quad \vec{n} \in B(\vec{k})
\end{aligned}
$$


It follows from the concavity of $\eta(x)=-x \log x$ and (4.25) that

$$
\begin{aligned}
S\left(\left.\omega\right|_{\Lambda(L \vec{k})}\right) \leq & \sum_{\substack{I_{\vec{n}} \in\{0,1\}^{N} \\
\vec{n} \in B(\vec{k})}} \eta\left(\omega\left(\prod_{\vec{n} \in B(\vec{k})}\left(\theta^{L \vec{n}}\left(P_{I_{\vec{n}}}\right)\right)\right)\right) \\
& +\sum_{\substack{J_{\vec{n}} \subset I(>N) \\
\vec{n} \in B(\vec{k})}} \eta\left(\omega\left(\prod_{\vec{n} \in B(\vec{k})}\left(\theta^{L \vec{n}}\left(P_{J_{\vec{n}}}\right)\right)\right)\right) \\
= & E(\Lambda, N, \vec{k})+\tilde{E}(\Lambda, N, \vec{k}),
\end{aligned}
$$

where $\tilde{E}(\Lambda, N, \vec{k})$ is defined to be the second term in the r.h.s. of (4.28). By using the concavity of $\eta(x)$ again, it follows that

$$
\tilde{E}(\Lambda, N, \vec{k}) \leq\left(k_{1} \ldots k_{\nu}\right) \sum_{J \subset I(>N)} \eta\left(\omega\left(P_{J}\right)\right) .
$$

Let $\left\{\gamma_{k}\right\}$ be the eigenvalues of the operator $h_{\Lambda}$ defined in (4.7). Let $r_{j} \equiv e^{-\gamma_{j}} /(1+$ $\left.e^{-\gamma_{j}}\right)$. By (4.24) and (3.6), it can be checked that

$$
\omega\left(P_{J}\right)=\prod_{j \in J} r_{j} \prod_{k \in J^{c}}\left(1-r_{k}\right) .
$$

Thus it follows that

$$
\sum_{J \subset I(>N)} \eta\left(\omega\left(P_{J}\right)\right)=-\sum_{J} \prod_{i \in J} r_{i} \prod_{j \in J^{c}}\left(1-r_{\jmath}\right)\left[\sum_{k \in J} \log r_{k}+\sum_{l \in J^{c}} \log \left(1-r_{l}\right)\right] .
$$

Following the procedure used in [20], we obtain

$$
\sum_{J \subset I(>N)} \eta\left(\omega\left(P_{J}\right)\right)=-\sum_{i=N+1}^{\infty} r_{\imath} \log r_{\imath}-\sum_{\imath=N+1}^{\infty}\left(1-r_{i}\right) \log \left(1-r_{\imath}\right) .
$$

Since we know that $[20]$ (see also Appendix)

$$
S\left(\left.\omega\right|_{, \Lambda}\right)=-\sum_{\imath=1}^{\infty} r_{\imath} \log r_{i}-\sum_{i=1}^{\infty}\left(1-r_{i}\right) \log \left(1-r_{\imath}\right)
$$

we conclude that

$$
\sum_{J \subset I(>N)} \eta\left(\omega\left(P_{J}\right)\right) \rightarrow 0 \quad \text { as } \quad N \rightarrow \infty
$$

The proposition follows from (4.28), (4.29) and the above convergence.

(b) The case of the CCR algebra: As before, let $\left\{Q_{i}(n): n=0,1,2, \ldots\right\}$ be the spectral projections of $N\left(f_{i}\right), i=1,2, \ldots$. Let $\mathbb{Z}_{+}=\{0,1,2, \ldots\}$. Denote by $\mathscr{T}\left(\mathbb{Z}_{+} ; I(>N)\right)$ the set of multi-indices $J \in \mathbb{Z}_{+}^{I(>N)}$ which have all but finite number of elements zero. For multi-indices

$$
I=\left(i_{1}, i_{2}, \ldots, i_{N}\right) \in \mathbb{Z}_{+}^{I(\leq N)}, \quad J=\left(j_{N+1}, j_{N+2}, \ldots\right) \in \mathscr{T}\left(\mathbb{Z}_{+} ; I(>N)\right),
$$

denote by

$$
Q_{I}=\prod_{\imath_{k} \in I} Q_{k}\left(i_{k}\right), \quad Q_{J}=\prod_{\jmath_{l} \in J} Q_{l}\left(j_{l}\right)
$$


Define the density operator $\hat{\varrho}_{\Lambda(L \vec{k})}$ on $\widetilde{F}_{\Lambda(L \vec{k})}$ by

$$
\begin{aligned}
& \hat{\varrho}_{\Lambda(L \vec{k})}=\sum_{\substack{I_{\vec{n}} \in \mathbb{Z}^{I(\leq N)} \\
\vec{n} \in B(\vec{k})}} \sum_{\substack{J_{\vec{n}} \in \mathcal{T}\left(\mathbb{Z}_{+} ; I(>N)\right) \\
\vec{n} \in B(\vec{k})}} \omega\left(\prod_{\vec{n} \in B(\vec{k})}\left(\theta^{L \vec{k}}\left(Q_{I_{\vec{n}}} Q_{J_{\vec{n}}}\right)\right)\right) \\
& \times \prod_{\vec{n} \in B(\vec{k})} \theta^{L \vec{n}}\left(Q_{I_{\vec{n}}} Q_{J_{\vec{n}}}\right) .
\end{aligned}
$$

We then follow the procedure used to obtain (4.28) and (4.29) for the CAR algebra to obtian that

$$
S\left(\omega_{\Lambda(L \vec{k})}\right) \leq I_{1}+I_{2}
$$

where

$$
\begin{gathered}
I_{1}=\sum_{I_{\vec{n}} \in \mathbb{Z}_{+}^{I \leq N)}} \eta\left(\omega\left(\prod_{\vec{n} \in B(\vec{k})}\left(\theta^{L \vec{n}}\left(Q_{I_{\vec{n}}}\right)\right)\right)\right), \\
I_{2}=\left(k_{1} \ldots k_{\nu}\right) \sum_{J \in \mathscr{T}\left(\mathbb{Z}_{+} ; I(>N)\right)} \eta\left(\omega\left(Q_{J}\right)\right) .
\end{gathered}
$$

We assert that

$$
I_{1} \leq E(\Lambda, N, \vec{k})+\left(k_{1} \ldots k_{\nu}\right) E^{\prime}(\Lambda, N)
$$

where $E(\Lambda, N, \vec{k})$ has been defined in (4.21), and

$$
E^{\prime}(\Lambda, N) \equiv \sum_{j=1}^{N} \sum_{k=N}^{\infty} \eta\left(\omega\left(Q_{\jmath}(k)\right)\right)
$$

In order to show the idea of the proof of our assertion, consider

$$
\tilde{I} \equiv \sum_{i=0}^{\infty} \sum_{j=0}^{\infty} \eta\left(\omega\left(Q_{1}(i) Q_{2}(j)\right)\right)
$$

Using the concavity of $\eta(x)$ and the Jensen's inequality, it can be checked that

$$
\begin{aligned}
\sum_{j=0}^{\infty} & \sum_{i=N}^{\infty} \eta\left(\omega\left(Q_{1}(i) Q_{2}(j)\right)\right) \\
\quad & \sum_{i=N}^{\infty} \eta\left(\omega\left(Q_{1}(i)\right)\right)-\sum_{j=0}^{\infty} \sum_{i=N}^{\infty} \omega\left(Q_{1}(i) Q_{2}(j)\right) \log \left(\omega\left(Q_{1}(i) Q_{2}(j)\right) / \omega\left(Q_{1}(i)\right)\right) \\
& \leq \sum_{i=N}^{\infty} \eta\left(\omega\left(Q_{1}(i)\right)\right)+\sum_{j=0}^{\infty} \eta\left(\omega\left(P_{1}(N) Q_{2}(j)\right)\right)-\eta\left(\omega\left(P_{1}(N)\right)\right),
\end{aligned}
$$

where $P_{1}(N)=\sum_{i=N}^{\infty} Q_{1}(i)$. See the notation in (4.14). Thus $\tilde{I}$ is bounded by

$$
\tilde{I} \leq \sum_{i=0}^{N} \sum_{j=0}^{\infty} \eta\left(\omega\left(P_{1}(i) Q_{2}(j)\right)\right)+\sum_{k=N}^{\infty} \eta\left(\omega\left(Q_{1}(k)\right)\right) .
$$


Employing the procedure used in the above again, we conclude that

$$
\tilde{I} \leq \sum_{i=0}^{N} \sum_{j=0}^{N} \eta\left(\omega\left(P_{1}(i) P_{2}(j)\right)\right)+\sum_{\imath=1}^{2} \sum_{k=N}^{\infty} \eta\left(\omega\left(Q_{\imath}(k)\right)\right) .
$$

By using the method employed in the above $\left(k_{1} \ldots k_{\nu}\right) N$-times, we proved the assertion in (4.34).

We next consider $I_{2}$ and $E^{\prime}(\Lambda, N)$ defined in (4.33) and (4.35) respectively. Notice that for any multi-index $I^{\prime}=\left(i_{1}, i_{2}, \ldots\right) \in \mathbb{Z}_{+}^{\mathbb{N}}$,

$$
\omega\left(Q_{I^{\prime}}\right)=\prod_{\imath_{k} \in I^{\prime}} \omega\left(Q_{k}\left(i_{k}\right)\right) \text {. }
$$

Thus it follows that

$$
I_{2}=\left(k_{1} \ldots k_{\nu}\right) \sum_{k=N+1}^{\infty}\left[\sum_{\imath_{k}=0}^{\infty} \eta\left(\omega\left(Q_{k}\left(i_{k}\right)\right)\right)\right] .
$$

A direct computation shows that

$$
S\left(\omega_{\Lambda}\right)=\sum_{k=1}^{\infty}\left[\sum_{\imath_{k}=0}^{\infty} \eta\left(\omega\left(Q_{k}\left(i_{k}\right)\right)\right)\right] .
$$

Thus it follows that $I_{2} \rightarrow 0$ as $N \rightarrow \infty$. From (4.35) and (4.36) it also follows that $E^{\prime}(\Lambda, N) \rightarrow 0$ as $N \rightarrow \infty$. The proposition now follows from (4.32), (4.34) and the above conclusions. Q.E.D.

\section{Basic Estimates}

In this section we derive some basic estimates which will be used in the sequel. As before, let $\chi_{\Lambda}$ be the projection operator from $L^{2}\left(\mathbb{R}^{\nu}\right)$ onto $L^{2}(\Lambda)$ and let $\sigma_{t}$ (resp. $\sigma_{t}^{\Lambda}$ ) be the modular automorphism of $\omega$ (resp. $\left.\omega_{\Lambda}=\left.\omega\right|_{\Lambda_{\Lambda}}\right)$. Recall the definitions of $B$ and $B^{\Lambda}$ in (3.7) and (4.6) respectively:

$$
B=A /(1+\sigma A), \quad B^{\Lambda}=A_{\Lambda} /\left(1+\sigma A_{\Lambda}\right) .
$$

Recall also the result in (4.8):

$$
B=e^{-h}, \quad B^{\Lambda}=e^{-h_{\Lambda}} .
$$

According to the notation in (3.18), we write that

$$
B_{\Lambda} \equiv \chi_{\Lambda} B \chi_{\Lambda}=\chi_{\Lambda} e^{-h} \chi_{\Lambda} .
$$

We first have the following result:

Lemma 5.1. For any bounded region $\Lambda \subset \mathbb{R}^{\nu}$, the relation

$$
B^{\Lambda}=\chi_{\Lambda} B^{1 / 2}\left[1-\sigma B^{1 / 2} \chi_{\Lambda}^{\perp} B^{1 / 2}\right]^{-1} B^{1 / 2} \chi_{\Lambda}
$$

holds, where $\sigma=+1$ (resp. $\sigma=-1)$ stand for the case of the CCR (resp. CAR) algebra. 
Proof. The above result was stated in [7] without detailed proof. We produce the proof. For any $f_{i}, g_{\jmath} \in L^{2}(\Lambda), i, j=1,2, \ldots, n$, denote by

$$
G=\prod_{j=1}^{n} a^{*}\left(f_{\jmath}\right) \prod_{\jmath=1}^{n-1} a\left(g_{\jmath}\right) .
$$

Then by the KMS condition $\omega(x y)=\omega\left(\sigma_{\imath}(y) x\right)$ and the fact that $\sigma_{\imath}(a(g))=a(B g)$, one has that

$$
\begin{aligned}
\omega\left(G a\left(g_{n}\right)\right) & =\omega\left(\sigma_{\imath}\left(a\left(g_{n}\right)\right) G\right) \\
& =\omega\left(a\left(B g_{n}\right) G\right) \\
& =\omega\left(a\left(\chi_{\Lambda} B g_{n}\right) G\right)+\omega\left(a\left(\chi_{\Lambda}^{\perp} B g_{n}\right) G\right) \\
& =\omega\left(a\left(\chi_{\Lambda} B g_{n}\right) G\right)+\sigma \omega\left(G a\left(\chi_{\Lambda}^{\perp} B g_{n}\right)\right) .
\end{aligned}
$$

Here we have used the CCRs and the CARs to get the last inequality. From the method used above, it follows that

$$
\omega\left(G a\left(\chi_{\Lambda}^{\perp} B g_{n}\right)\right)=\omega\left(a\left(\chi_{\Lambda} B \chi_{\Lambda}^{\perp} B g_{n}\right) G\right)+\sigma \omega\left(G a\left(\chi_{\Lambda}^{\perp} B \chi_{\Lambda}^{\perp} B g_{n}\right)\right) .
$$

Iterating the above process, one has that

$$
\begin{aligned}
\omega\left(G a\left(g_{n}\right)\right)= & \omega\left(a\left(\chi_{\Lambda} B \chi_{\Lambda} g_{n}\right) G\right) \\
& +\sigma \omega\left(a\left(\chi_{\Lambda} B \chi_{\Lambda}^{\perp} B \chi_{\Lambda} g_{n}\right) G\right) \\
& \cdots \\
& +\sigma^{n} \omega\left(a\left(\chi_{\Lambda} B \chi_{\Lambda}^{\perp} B \ldots \chi_{\Lambda}^{\frac{1}{\Lambda}} B \chi_{\Lambda} g_{n}\right) G\right) \\
& \cdots \\
= & \omega\left(a\left(\chi_{\Lambda} B^{1 / 2}\left[1-\sigma B^{1 / 2} \chi_{\Lambda}^{\perp} B^{1 / 2}\right]^{-1} B^{1 / 2} \chi_{\Lambda} g_{n}\right) G\right) .
\end{aligned}
$$

Here we have used Assumption 3.1(a) and (b) to show the convergence. The above relation holds for any $g_{n} \in L^{2}(\Lambda)$. On the other hand, by the KMS condition for $\sigma_{t}^{\Lambda}$ we have that $\omega\left(G a\left(g_{n}\right)\right)=\omega\left(a\left(B^{\Lambda} g_{n}\right) G\right)$ for any $G \in \mathscr{b}_{\Lambda}, g_{n} \in L^{2}(\Lambda)$. This proved the lemma completely. Q.E.D.

We next derive some estimates on the operators $B_{\Lambda}$ and $B^{\Lambda}$ defined in (5.1) and (4.6) respectively:

Lemma 5.2. There are constants $c_{1}, c_{2}$ and $c_{3}$ independent of $\Lambda$ such that the bounds

$$
\begin{aligned}
& \operatorname{Tr}\left(B_{\Lambda}\right) \leq c_{1}|\Lambda|, \quad \operatorname{Tr}\left(B^{\Lambda}\right) \leq c_{2}|\Lambda|, \\
& \operatorname{Tr}\left(\chi_{\Lambda}\left(B^{1 / 2}-\left(B^{\Lambda}\right)^{1 / 2}\right)^{2} \chi_{\Lambda}\right) \leq c_{3}|\partial \Lambda|
\end{aligned}
$$

hold for any $\Lambda=(-L / 2, L / 2)^{\nu}$, where $\operatorname{Tr}$ is the trace on $L^{2}(\Lambda)$, and $\partial \Lambda$ the boundary of $\Lambda$.

Proof. (a) The case of the CCR algebra: Assumption 3.1(b) implies that $\hat{K}_{B}$ is integrable and so the first bound follows from the result analogous to that in (3.19). Lemma 5.1 implies that

$$
B^{\Lambda} \leq B_{\Lambda}
$$

Thus the second inequality follows from (5.2) and the first inequality in the lemma. We next prove the last inequality. Notice that

$$
\left(\chi_{\Lambda} B^{1 / 2} \chi_{\Lambda}\right)^{2} \leq B_{\Lambda}
$$


From (5.2), (5.3) and the fact that $0 \leq A \leq B \Rightarrow 0 \leq A^{1 / 2} \leq B^{1 / 2}$, it follows that

$$
\left(B^{\Lambda}\right)^{1 / 2} \leq B_{\Lambda}^{1 / 2}
$$

and

$$
\chi_{\Lambda} B^{1 / 2} \chi_{\Lambda} \leq B_{\Lambda}^{1 / 2}
$$

Denote by $S(\Lambda)$ the term in the 1.h.s. of the inequality in the lemma:

$$
S(\Lambda)=\operatorname{Tr}\left(\chi_{\Lambda}\left(B^{1 / 2}-\left(B^{\Lambda}\right)^{1 / 2}\right)^{2} \chi_{\Lambda}\right) .
$$

Notice that

$$
S(\Lambda)=\operatorname{Tr}\left(B_{\Lambda}+B^{\Lambda}\right)-2 \operatorname{Tr}\left(\chi_{\Lambda} B^{1 / 2} \chi_{\Lambda}\left(B^{\Lambda}\right)^{1 / 2} \chi_{\Lambda}\right) .
$$

Using (5.4) and (5.5) we obtain that

$$
\begin{array}{rlr}
\operatorname{Tr}\left(\chi_{\Lambda} B^{1 / 2} \chi_{\Lambda}\left(B^{\Lambda}\right)^{1 / 2} \chi_{\Lambda}\right) & =-\operatorname{Tr}\left(\left(B_{\Lambda}^{1 / 2}-\chi_{\Lambda} B^{1 / 2} \chi_{\Lambda}\right)\left(B^{\Lambda}\right)^{1 / 2}\right)+\operatorname{Tr}\left(B_{\Lambda}^{1 / 2}\left(B^{\Lambda}\right)^{1 / 2}\right) \\
& \geq-\operatorname{Tr}\left(\left(B_{\Lambda}^{1 / 2}-\chi_{\Lambda} B^{1 / 2} \chi_{\Lambda}\right) B_{\Lambda}^{1 / 2}\right)+\operatorname{Tr}\left(B^{\Lambda}\right) & \text { by (5.4) } \\
& \geq-\operatorname{Tr}\left(B_{\Lambda}-\left(\chi_{\Lambda} B^{1 / 2} \chi_{\Lambda}\right)^{2}\right)+\operatorname{Tr}\left(B^{\Lambda}\right) . & \text { by (5.5) }
\end{array}
$$

Thus from (5.6) and the above bound it follows that

$$
S(\Lambda) \leq \operatorname{Tr}\left(B_{\Lambda}-B^{\Lambda}\right)+2 \operatorname{Tr}\left(B_{\Lambda}-\left(\chi_{\Lambda} B^{1 / 2} \chi_{\Lambda}\right)^{2}\right) .
$$

Using Lemma 5.1, the fact that

$$
B_{\Lambda}=\left(\chi_{\Lambda} B^{1 / 2} \chi_{\Lambda}\right)^{2}+\chi_{\Lambda} B^{1 / 2} \chi_{\Lambda}^{\perp} B^{1 / 2} \chi_{\Lambda},
$$

Assumption 3.1(c) and Remark 3.2(d), we conclude that

$$
\begin{aligned}
S(\Lambda) \leq & \operatorname{Tr}\left(\chi_{\Lambda} B \chi_{\Lambda}^{\perp} B \chi_{\Lambda}\right)+2 \operatorname{Tr}\left(\chi_{\Lambda} B^{1 / 2} \chi_{\Lambda}^{\perp} B^{1 / 2} \chi_{\Lambda}\right) \\
\leq & c d_{\Lambda}^{\nu} x \int_{\Lambda^{c}} d^{\nu} y \prod_{j=1}^{\nu}\left(1+\left|x_{\jmath}-y_{\jmath}\right|\right)^{-2(1+\alpha)} \\
\leq & c \sum_{k=1}^{n} a_{k}\left(\int_{-L / 2}^{L / 2} d x \int_{L / 2}^{\infty} d y\left(1+|x-y|^{-2(1+\alpha)}\right)^{k}\right. \\
& \times\left(\int_{-L / 2}^{L / 2} d x \int_{-L / 2}^{L / 2} d y\left(1+|x-y|^{-2(1+\alpha)}\right)^{n-k}\right. \\
\leq & c^{\prime} L^{\nu-1} .
\end{aligned}
$$

Here we have decomposed $\Lambda^{c}$ into several regions to obtain the third inequality. This proves the last inequality for the CAR algebra.

(b) The case of the CCR algebra: The first inequality follows from the argument for the CAR algebra. The second inequality follows from (4.6) and Remark 3.2(b). Recall the notation in (5.6). Lemma 5.1 implies that

$$
B^{\Lambda} \geq B_{\Lambda}
$$


and so from (5.7), the above bound and (5.5), it follows that

$$
\begin{aligned}
S(\Lambda) & \leq \operatorname{Tr}\left(B_{\Lambda}+B^{\Lambda}\right)-2 \operatorname{Tr}\left(\left(\chi_{\Lambda} B^{1 / 2} \chi_{\Lambda}\right)^{2}\right) \\
& =\operatorname{Tr}\left(B^{\Lambda}-B_{\Lambda}\right)+2 \operatorname{Tr}\left(\chi_{\Lambda} B^{1 / 2} \chi_{\Lambda}^{\perp} B^{1 / 2} \chi_{\Lambda}\right) .
\end{aligned}
$$

We now use Lemma 5.1 and the fact that $\left\|B^{1 / 2} \chi_{\Lambda} B^{1 / 2}\right\| \leq M_{1}<1$ by Assumption 3.1(a) to conclude that

$$
S(\Lambda) \leq c \operatorname{Tr}\left(\chi_{\Lambda} B \chi_{\Lambda}^{\perp} B \chi_{\Lambda}\right)+2 \operatorname{Tr}\left(\chi_{\Lambda} B^{1 / 2} \chi_{\Lambda}^{\perp} B^{1 / 2} \chi_{\Lambda}\right)
$$

for some constant $c$ independent of $\Lambda$. As in the case of the CAR, the last inequality follows from Assumption 3.1(c) and Remark 3.2(d), and the above bound. Q.E.D.

Recall the definitions of the operators $B^{\Lambda}$ and $h_{\Lambda}$ in (4.6) and (4.7) respectively. By (4.8) these operators are related as $B^{\Lambda}=\exp \left(-h_{\Lambda}\right)$.

Lemma 5.3. Let $\Lambda=(-L / 2, L / 2)^{\nu} \subset \mathbb{R}^{\nu}$. Then there exists a constant $c$ independent of $\Lambda$ such that the bound

$$
\left|\operatorname{Tr}\left(h_{\Lambda} B^{\Lambda}\right)\right| \leq c|\Lambda|
$$

holds, where $\operatorname{Tr}$ is the trace on $L^{2}(\Lambda)$.

Proof. Let $\left\{g_{n}: n=\left(n_{1}, \ldots, n_{\nu}\right) \in \mathbb{Z}^{\nu}\right\}$ be the orthogonal basis of $L^{2}(\Lambda)$ given by

$$
g_{n}(x)=\prod_{j=1}^{\nu}\left\{L^{-1 / 2} \exp \left(i 2 \pi n_{j} x_{j} / L\right)\right\}
$$

and let $\Delta_{\Lambda, P}$ be the Laplacian operator on $L^{2}(\Lambda)$ with periodic boundary conditions on $\partial \Delta$. It follows that

$$
-\Delta_{\Lambda, P} g_{n}=\left|\frac{2 \pi n}{L}\right|^{2} g_{n}
$$

where $|n|^{2}=n_{1}^{2}+\ldots+n_{\nu}^{2}$. Let $\alpha$ be the constant in Assumption 3.1(b). For given (fixed) constant $\alpha^{\prime}$ satisfying $0<2 \alpha^{\prime}<\alpha<1$, we denote by

$$
D_{\Lambda} \equiv \exp \left\{-\left(-\Delta_{\Lambda, P}\right)^{\alpha^{\prime}}\right\} \text {. }
$$

Using the inequality (4.27) we obtain that

$$
\begin{aligned}
\operatorname{Tr}\left(h_{\Lambda} B^{\Lambda}\right) & =-\operatorname{Tr}\left(B^{\Lambda} \log \left(B^{\Lambda}\right)\right) \\
& =-\operatorname{Tr}\left[B^{\Lambda}\left(\log \left(B^{\Lambda}\right)-\log \left(D_{\Lambda}\right)\right)\right]-\operatorname{Tr}\left(B^{\Lambda} \log \left(D_{\Lambda}\right)\right) \\
& \left.\leq \operatorname{Tr}\left(D_{\Lambda}-B^{\Lambda}\right)+\operatorname{Tr}\left(\left(-\Delta_{\Lambda, P}\right)^{\alpha^{\prime}} B^{\Lambda}\right)\right) .
\end{aligned}
$$

Using Lemma 5.2 and (5.9), it can be checked that

$$
\left|\operatorname{Tr}\left(D_{\Lambda}-B^{\Lambda}\right)\right| \leq c_{1}\left(\alpha^{\prime}\right)|\Lambda|
$$

for some constant $c_{1}\left(\alpha^{\prime}\right)$ independent of $\Lambda$.

We denote for any $n \in \mathbb{Z}^{\nu}$,

$$
\hat{\chi}_{\Lambda, n}(k) \equiv \prod_{j=1}^{\nu}\left\{\frac{2}{\sqrt{2 \pi L}} \sin \left(\frac{L}{2}\left(k_{j}-\frac{2 \pi n_{\jmath}}{L}\right)\right) /\left(k_{j}-\frac{2 \pi n_{j}}{L}\right)\right\} .
$$


Since by (4.6) and Assumption 3.1(a) one has that $B^{\Lambda} \leq c^{\prime} A_{\Lambda}$ for some constant $c^{\prime}$ independently of $\Lambda$, it follows that

$$
\operatorname{Tr}\left(\left(-\Delta_{\Lambda, P}\right)^{\alpha^{\prime}} B^{\Lambda}\right) \leq c^{\prime} \operatorname{Tr}\left(\left(-\Delta_{\Lambda, P}\right)^{\alpha^{\prime}} A_{\Lambda}\right) .
$$

On the other hand it follows from (3.11), (3.12) and (5.9) that

$$
\operatorname{Tr}\left(\left(-\Delta_{\Lambda, P}\right)^{\alpha^{\prime}} A_{\Lambda}\right)=\sum_{n \in \mathbb{Z}^{\nu}}\left|\frac{2 \pi n}{L}\right|^{2 \alpha^{\prime}} \int\left(\hat{\chi}_{\Lambda, n}(k)\right)^{2} \hat{K}_{A}(k) d^{\nu} k
$$

Using Assumption 3.1(a) and (b), and the fact that

$$
\left|\hat{\chi}_{\Lambda, n}(k)\right| \leq c \prod_{j=1}^{\nu}\left(1+\left|k_{j}-2 \pi n_{j} / L\right|\right)^{-1}
$$

for some constant $c$ independent of $\Lambda$, it is easy to show that

$$
\int\left(\hat{\chi}_{\Lambda}(k)\right)^{2} \hat{K}_{A}(k) d^{\nu} k \leq M \prod_{j=1}^{\nu}\left(1+\left|2 \pi n_{j} / L\right|\right)^{-(1+\alpha)}
$$

for some constant $M$ independent of $\Lambda$. From (5.14), (5.15) and the above bound, we conclude that

$$
\operatorname{Tr}\left(\left(-\Delta_{\Lambda, P}\right)^{\alpha^{\prime}} B^{\Lambda}\right) \leq c_{2}\left(\alpha^{\prime}\right)|\Lambda| .
$$

The lemma follows from (5.11), (5.12) and the above bound. Q.E.D.

From Lemma 5.3 and Assumption 3.1(a) and (b) we get the following result:

Lemma 5.4. Let $\Lambda$ be as in Lemma 5.3. Then there exists a constant c independent of $\Lambda$ such that the bound

holds.

$$
\left|\operatorname{Tr}\left(h_{\Lambda}^{3 / 2} B^{\Lambda}\right)\right| \leq c|\Lambda|
$$

Proof. Since $B^{\Lambda}=\exp \left(-h_{\Lambda}\right)$, we have that

$$
\operatorname{Tr}\left(h_{\Lambda}^{3 / 2} B^{\Lambda}\right)=-2 \operatorname{Tr}\left(h_{\Lambda}^{1 / 2} B^{\Lambda} \log \left(\left(B^{\Lambda}\right)^{1 / 2}\right)\right) .
$$

Let $D_{\Lambda}$ be defined as in (5.10). From the above, it follows that

$$
\begin{aligned}
\operatorname{Tr}\left(h_{\Lambda}^{3 / 2} B^{\Lambda}\right)= & -2 \operatorname{Tr}\left(h_{\Lambda}^{1 / 2} B^{\Lambda}\left[\log \left(\left(B^{\Lambda}\right)^{1 / 2}-\log \left(\left(B^{\Lambda}\right)^{1 / 2}+D_{\Lambda}^{1 / 2}\right)\right]\right)\right. \\
& -2 \operatorname{Tr}\left(h_{\Lambda}^{1 / 2} B^{\Lambda} \log \left(\left(B^{\Lambda}\right)^{1 / 2}+D_{\Lambda}^{1 / 2}\right)\right) \\
\equiv & E_{\Lambda, 1}+E_{\Lambda, 2} .
\end{aligned}
$$

As before, let $\left\{\gamma_{k}\right\}$ be the eigenvalues of $h_{\Lambda}$ and $\left\{f_{k}\right\}$ the corresponding normalized eigenvectors. Then we have that

$$
E_{\Lambda, 1}=-2 \sum_{k=1}^{\infty}\left(\gamma_{k}^{1 / 2} e^{-\gamma_{k}}\left[\log \left(e^{-\gamma_{k} / 2}\right)-\left(f_{k}, \log \left(e^{-h_{\Lambda} / 2}+D_{\Lambda}^{1 / 2}\right) f_{k}\right)\right]\right)
$$

By Jensen's inequality we obtain that

$$
\left(f_{k}, \log \left(e^{-h_{\Lambda} / 2}+D_{\Lambda}^{1 / 2}\right) f_{k}\right) \leq \log \left(e^{-\gamma_{k} / 2}+\left(f_{k}, D_{\Lambda}^{1 / 2} f_{k}\right)\right) .
$$


By taking $x=\exp \left(-\gamma_{k} / 2\right), y=\left(f_{k}, D_{\Lambda}^{1 / 2} f_{k}\right)$ and using the fact that $-x(\log x-$ $\log (x+y)) \leq y$ for any positive $x$ and $y$, we obtain that

$$
\begin{aligned}
E_{\Lambda, 1} & \leq 2 \sum_{k=1}^{\infty}\left(\gamma_{k}^{1 / 2} e^{-\gamma_{k} / 2}\left(f_{k}, D_{\Lambda}^{1 / 2} f_{k}\right)\right) \\
& =2 \operatorname{Tr}\left(h_{\Lambda}^{1 / 2}\left(B^{\Lambda}\right)^{1 / 2} D_{\Lambda}^{1 / 2}\right) \\
& \leq 2\left(\operatorname{Tr}\left(h_{\Lambda}\left(B^{\Lambda}\right)\right)\right)^{1 / 2}\left(\operatorname{Tr}\left(D_{\Lambda}\right)\right)^{1 / 2}
\end{aligned}
$$

Thus from Lemma 5.3 and the fact that $\operatorname{Tr}\left(D_{\Lambda}\right) \leq c|\Lambda|$ for some constant $c$ independent of $\Lambda$, it follows that the bound

$$
E_{\Lambda, 1} \leq c^{\prime}|\Lambda|
$$

holds uniformly in $\Lambda$.

We next consider $E_{\Lambda, 2}$ defined in (5.17). Notice that

$$
\begin{aligned}
E_{\Lambda, 2} & \leq-2 \operatorname{Tr}\left(h_{\Lambda}^{1 / 2} B^{\Lambda} \log \left(D_{\Lambda}^{1 / 2}\right)\right) \\
& =\operatorname{Tr}\left(h_{\Lambda}^{1 / 2} B^{\Lambda}\left(-\Delta_{\Lambda, P}\right)^{\alpha^{\prime}}\right) \\
& \leq\left(\operatorname{Tr}\left(h_{\Lambda}\left(B^{\Lambda}\right)\right)\right)^{1 / 2}\left(\operatorname{Tr}\left(\left(-\Delta_{\Lambda, P}\right)^{2 \alpha^{\prime}} B^{\Lambda}\right)\right)^{1 / 2} .
\end{aligned}
$$

We choose $\alpha^{\prime}$ so that $0<4 \alpha^{\prime}<\alpha$. Then by using the same method as that in the proof of (5.16) we get that

$$
\left.\operatorname{Tr}\left(\left(-\Delta_{\Lambda, P}\right)^{2 \alpha^{\prime}} B^{\Lambda}\right)\right) \leq c|\Lambda|
$$

for some constant $c$. Thus from Lemma 5.3 and the above bound we conclude that

$$
E_{\Lambda, 2} \leq c^{\prime \prime}|\Lambda|
$$

uniformly in $\Lambda$. The lemma follows from $(5.17),(5.18)$ and the above bound. Q.E.D.

\section{Control of Entropy Defect: Proof of Proposition 4.3}

We are now in position to show that the average of the entropy defects tends to zero as $\Lambda$ tends to $\mathbb{R}^{\nu}$ (proposition 4.3) and so complete our main result (Theorem 3.3). Recall the entropy defect defined in (4.21):

$$
D(\Lambda, N)=\sum_{I} \omega\left(P_{I}\right) S\left(\left.\hat{\omega}_{I}\right|_{\Lambda} ^{N)}\right)
$$

where $\mathscr{A}_{\Lambda}^{(N)}\left(\subset \cdot \mathscr{A}_{\Lambda}\right)$ is the algebra generated by $\left\{P_{I}\right\}_{I}$, and

$$
\hat{\omega}_{I}(a)=\omega\left(\sigma_{\imath / 2}\left(P_{I}\right) a\right) / \omega\left(P_{I}\right) .
$$

See the definition of $P_{I}$ in (4.12) and (4.16).

We first describe briefly the reason why Proposition 4.3 should hold. Let $\hat{\omega}_{I}^{\Lambda}$ be the state defined by replacing $\sigma_{i / 2}$ with $\sigma_{i / 2}^{\Lambda}$ in (6.1). Then one may check that $S\left(\left.\hat{\omega}_{I}^{\Lambda}\right|_{\Lambda} ^{(N)}\right)=0$, and so the corresponding entropy defect equals zero. One may expect that the difference, $\left(\hat{\omega}_{I}-\hat{\omega}_{I}^{\Lambda}\right)(a), a \in A_{\Lambda}^{(N)}$, should be bounded by a term which depends only on the surface $\partial \Lambda$ of $\Lambda$. 
The proof of Proposition 4.3 will consist of several steps. Let us try to make the entropy defect $D(\Lambda, N)$ bounded by a sum of terms which can be estimated eventually. Recall that $\mathscr{b}_{\Lambda}^{(N)}$ is the finite dimensional abelian algebra generated by $\left\{P_{I}\right\}_{I}$. Thus $\mathscr{A}_{\Lambda}^{(N)}$ can be represented by an abelian algebra on a finite dimensional Hilbert space $\mathscr{H}_{\Lambda}^{(N)}\left(\cong \mathbb{C}^{M}\right)$ such that $\operatorname{Tr}_{\mathscr{H}_{\Lambda}^{(N)}}\left(P_{I}\right)=1$. Denote by $\varrho_{\Lambda, I}^{(N)}$ the density operator on $\mathscr{H}_{\Lambda}^{(N)}$ corresponding to the state $\left.\hat{\omega}_{I}\right|_{\mathscr{C}_{\Lambda}^{(N)}}$. Then for any $a \in \mathscr{A}_{\Lambda}^{(N)}$,

$$
\operatorname{Tr}_{\mathscr{H}_{\Lambda}^{(N)}}\left(\varrho_{\Lambda, I}^{(N)} a\right)=\omega\left(\sigma_{i / 2}\left(P_{I}\right) a\right) / \omega\left(P_{I}\right)=\sum_{I^{\prime}} \operatorname{Tr}_{\mathscr{H}_{\Lambda}^{(N)}}\left(\varrho_{\Lambda, I}^{(N)} a P_{I^{\prime}}\right)
$$

and

$$
D(\Lambda, N)=-\sum_{I} \omega\left(P_{I}\right) \operatorname{Tr}_{\mathscr{H}_{\Lambda}^{(N)}}\left(\varrho_{\Lambda, I}^{(N)} \log \left(\varrho_{\Lambda, I}^{(N)}\right)\right) .
$$

From now on, we suppress $\mathscr{H}_{\Lambda}^{(N)}$ in the notation $\operatorname{Tr}_{\mathscr{H}}{ }_{\Lambda}^{(N)}$ for notational simplification. For a given multi-index $I\left(I \in\{0,1\}^{N}\right.$ for the CAR algebra and $I \in\{0,1, \ldots, N\}^{N}$ for the CCR algebra), we define

$$
\tilde{\varrho}_{\Lambda, I}^{(N)} \equiv \sum_{I^{\prime}}\left\{\omega\left(\sigma_{i / 2}\left(P_{I}\right) P_{I^{\prime}}\right) / \omega\left(P_{I}\right)\right\} P_{I^{\prime}}=\sum_{I^{\prime}}\left\{\operatorname{Tr}\left(\varrho_{\Lambda, I}^{(N)} P_{I^{\prime}}\right)\right\} P_{I^{\prime}}
$$

on $\mathscr{H}_{\Lambda}^{(N)}$. Notice that $\operatorname{Tr}\left(\tilde{\varrho}_{\Lambda, I}^{(N)}\right)=1$ by the fact that $\operatorname{Tr}\left(P_{I^{\prime}}\right)=1$ for any multi-index $I^{\prime}$. Using the inequality (4.27) and the relation (6.2), we get

$$
\begin{aligned}
D_{\Lambda, N} & \leq-\sum_{I} \omega\left(P_{I}\right) \operatorname{Tr}\left(\varrho_{\Lambda, I}^{(N)} \log \left(\tilde{\varrho}_{\Lambda, I}^{(N)}\right)\right) \\
& =-\sum_{I} \sum_{I^{\prime}} \omega\left(P_{I}\right)\left[\frac{\omega\left(\sigma_{i / 2}\left(P_{I}\right) P_{I^{\prime}}\right)}{\omega\left(P_{I}\right)} \log \left(\frac{\omega\left(\sigma_{\imath / 2}\left(P_{I}\right) P_{I^{\prime}}\right)}{\omega\left(P_{I}\right)}\right)\right] \\
& =I_{\Lambda, N}+I I_{\Lambda, N},
\end{aligned}
$$

where

$$
\begin{gathered}
I_{\Lambda, N} \equiv \sum_{I} \omega\left(\sigma_{i / 2}\left(P_{I}\right) P_{I}\right) \log \left(\omega\left(\sigma_{i / 2}\left(P_{I}\right) P_{I}\right) / \omega\left(P_{I}\right)\right) \\
I I_{\Lambda, N} \equiv \sum_{I} \sum_{I^{\prime}:} \omega\left(\sigma_{\imath / 2}\left(P_{I}\right) P_{I^{\prime}}\right) \log \left(\omega\left(\sigma_{\imath / 2}\left(P_{I}\right) P_{I^{\prime}}\right) / \omega\left(P_{I}\right)\right) .
\end{gathered}
$$

We first consider $I_{\Lambda, N}$ defined in the above. We shall use the inequality

$$
-x(\log x-\log (x+y)) \leq y
$$

for any positive numbers $x$ and $y$. Take $x=\omega\left(\sigma_{i / 2}\left(P_{I}\right) P_{I}\right) / \omega\left(P_{I}\right)$ and $y=$ $\omega\left(\sigma_{i / 2}\left(P_{I}\right)\left(1-P_{I}\right)\right) / \omega\left(P_{I}\right)$. Notice that $x+y=1$. From inequality (6.7), it follows that $-x \log x=-x(\log x-\log (x+y))-x \log (x+y) \leq y$. Thus we conclude that the bound

$$
I_{\Lambda, N} \leq \sum_{I} \omega\left(\sigma_{i / 2}\left(P_{I}\right)\left(1-P_{I}\right)\right)
$$

holds. 
We next consider $I I_{\Lambda, N}$ defined in (6.6). For notational convenience, denote that for any subset $J \subset\{1,2, \ldots, N\}=I(\leq N)$,

$$
\begin{gathered}
\mathscr{T}(J)=\{0,1\}^{J} \quad(\mathrm{CAR}), \\
\mathscr{T}(J)=\{0,1, \ldots, N\}^{J} \quad(\mathrm{CCR}) .
\end{gathered}
$$

Let, $I, I^{\prime} \in \mathscr{T}(I(\leq N))$ such that $I \neq I^{\prime}\left(P_{I} \neq P_{I^{\prime}}\right)$. Then there exists a subset $J \subset\{1,2, \ldots, N\}=I(\leq N)$ with $J^{c} \neq \emptyset$ such that

$$
\begin{gathered}
P_{I}=P_{I_{1}} P_{I_{2}}, \quad P_{I^{\prime}}=P_{I_{1}} P_{I_{2}^{\prime}}, \\
I_{1} \in \mathscr{T}(J), \quad I_{2}, I_{2}^{\prime} \in \mathscr{T}\left(J^{c}\right),
\end{gathered}
$$

where for given $I_{2}=\left(\left(i_{k}\right): k \in J^{c}\right), I_{2}^{\prime}$ belongs to the following set:

$$
\begin{gathered}
\mathscr{T}\left(J^{c}: I_{2}\right)=\left\{\left(i_{k}^{\prime}\right): i_{k}^{\prime} \in\{0,1\}-\left\{i_{k}\right\}, k \in J^{c}\right\} \quad(\mathrm{CAR}), \\
\mathscr{T}\left(J^{c}: I_{2}\right)=\left\{\left(i_{k}^{\prime}\right): i_{k}^{\prime} \in\{0,1, \ldots, N\}-\left\{i_{k}\right\}, k \in J^{c}\right\} \quad(\mathrm{CCR}) .
\end{gathered}
$$

Notice that in the case of the CARs, $\mathscr{T}\left(J^{c}: I_{2}\right)$ is singleton. For instance, let $P_{I_{2}}=\prod_{k \in J^{c}} P_{k}\left(i_{k}\right)$. Then $P_{I_{2}^{\prime}}=\prod_{k \in J^{c}}\left(1-P_{k}\left(i_{k}\right)\right)$ in the case of the CARs. Thus the term $I I_{\Lambda, N}$ defined in (6.6) can be written as

$$
\begin{aligned}
I I_{\Lambda, N}= & -\sum_{\substack{J \subset I(\leq N): \\
J^{c} \neq \emptyset}} \sum_{\substack{I_{1} \in \mathscr{T}(J) \\
I_{2} \in \mathcal{T}\left(J^{c}\right)}} \sum_{I_{2}^{\prime} \in \mathscr{T}\left(J^{c}: I_{2}\right)} \\
& \times\left\{\omega\left(\sigma_{i / 2}\left(P_{I_{1}} P_{I_{2}}\right) P_{I_{1}} P_{I_{2}^{\prime}}\right) \log \left(\frac{\omega\left(\sigma_{i / 2}\left(P_{I_{1}} P_{I_{2}}\right) P_{I_{1}} P_{I_{2}^{\prime}}\right)}{\omega\left(P_{I_{1}} P_{I_{2}}\right)}\right)\right\} .
\end{aligned}
$$

As before, let $\left\{\gamma_{k}\right\}_{k=1}^{\infty}$ be the eigenvalues of $h_{\Lambda}$ and $\left\{f_{k}\right\}$ the corresponding eigenvectors. For any $J \subset I(\leq N)$, let $\mathscr{C}_{\Lambda, J}$ be the algebra generated by the spectral projections of $N\left(f_{k}\right), k \in J$. Define a state $\omega_{\Lambda, J}$ on $\mathscr{A}_{\Lambda, J}$ by

$$
\begin{gathered}
\omega_{\Lambda, J}\left(N\left(f_{k}\right)\right)=|\Lambda|^{-1} e^{-\gamma_{k}} /\left(1-\sigma|\Lambda|^{-1} e^{-\gamma_{k}}\right), k \in J, \\
\omega_{\Lambda, J}\left(\prod_{k \in J} N\left(f_{k}\right)\right)=\prod_{k \in J} \omega_{\Lambda, J}\left(N\left(f_{k}\right)\right),
\end{gathered}
$$

where $\sigma=+1$ (resp. $\sigma=-1$ ) stands for the CCR (resp. CAR) algebra. Define

$$
\begin{aligned}
I I_{\Lambda, N}^{(1)}= & -\sum_{\substack{J \subset I(\leq N): \\
J^{c} \neq \emptyset}} \sum_{\substack{I_{1} \in \mathscr{T}(J) \\
I_{2} \in \mathscr{T}\left(J^{c}\right)}} \sum_{I_{2}^{\prime} \in \mathscr{T}\left(J^{c}: I_{2}\right)} \\
& \times\left\{\omega\left(\sigma_{i / 2}\left(P_{I_{1}} P_{I_{2}}\right) P_{I_{1}} P_{I_{2}^{\prime}}\right) \log \left(\frac{\omega\left(\sigma_{i / 2}\left(P_{I_{1}} P_{I_{2}}\right) P_{I_{1}} P_{I_{2}^{\prime}}\right)}{\omega\left(P_{I_{1}} P_{I_{2}}\right) \omega_{\Lambda, J^{c}}\left(P_{I_{2}}\right)}\right)\right\} \\
I I_{\Lambda, N}^{(2)}= & -\sum_{\substack{J \subset I(\leq N): \\
J^{c} \neq \emptyset}} \sum_{\substack{I_{1} \in \mathscr{T}(J) \\
I_{2} \in \mathscr{T}\left(J^{c}\right)}} \sum_{I_{2}^{\prime} \in \mathscr{T}\left(J^{c}: I_{2}\right)} \\
& \times\left\{\omega\left(\sigma_{\imath / 2}\left(P_{I_{1}} P_{I_{2}}\right) P_{I_{1}} P_{I_{2}^{\prime}}\right) \log \left(\omega_{\Lambda, J^{c}}\left(P_{I_{2}}\right)\right)\right\} .
\end{aligned}
$$


The above quantities are defined so that the relation

$$
I I_{\Lambda, N}=I I_{\Lambda, N}^{(1)}+I I_{\Lambda, N}^{(2)}
$$

holds by (6.12).

We consider the term $I I_{\Lambda, N}^{(1)}$ defined in (6.14). Taking $x=\frac{\omega\left(\sigma_{i / 2}\left(P_{I_{1}} P_{I_{2}}\right) P_{I_{1}} P_{I_{2}^{\prime}}\right)}{\omega\left(P_{I_{1}} P_{I_{2}}\right) \omega_{\Lambda, J^{c}}\left(P_{I_{2}}\right)}$ and $y=\omega_{\Lambda, J^{c}}\left(P_{I_{2}^{\prime}}\right)$, and using the inequality (6.7) and $-\log (x+y) \leq-\log (y)$, we obtain that

$$
I I_{\Lambda, N}^{(1)} \leq I I_{\Lambda . N}^{(1.1)}+I I_{\Lambda, N}^{(1.2)}
$$

where

$$
\begin{aligned}
I I_{\Lambda, N}^{(1.1)} \equiv & \sum_{\substack{J \subset I(\leq N): \\
J^{c} \neq \emptyset}} \sum_{\substack{I_{1} \in \mathscr{T}(J) \\
I_{2} \in \mathscr{T}\left(J^{c}\right)}} \sum_{I_{2}^{\prime} \in \mathscr{T}\left(J^{c}: I_{2}\right)} \\
& \times \omega\left(P_{I_{1}} P_{\left.I_{2}\right)} \omega_{\Lambda, J^{c}}\left(P_{I_{2}}\right) \omega_{\Lambda, J^{c}}\left(P_{I_{2}^{\prime}}\right)\right. \\
I I_{\Lambda, N}^{(1.2)} \equiv & -\sum_{\substack{J \subset I(\leq N): \\
J^{c} \neq \emptyset}} \sum_{\substack{I_{1} \in \mathscr{T}(J) \\
I_{2} \in \mathscr{T}\left(J^{c}\right)}} \sum_{I_{2}^{\prime} \in \mathscr{T}\left(J^{c}: I_{2}\right)} \\
& \times\left\{\omega\left(\sigma_{i / 2}\left(P_{I_{1}} P_{I_{2}}\right) P_{I_{1}} P_{I_{2}^{\prime}}\right) \log \left(\omega_{\Lambda, J^{c}}\left(P_{I_{2}^{\prime}}\right)\right)\right\} .
\end{aligned}
$$

From (6.5), (6.15) and (6.16), it follows that the bound

holds.

$$
D(\Lambda, N) \leq I_{\Lambda, N}+\left(I I_{\Lambda, N}^{(1.1)}+I I_{\Lambda, N}^{(1.2)}\right)+I I_{\Lambda, N}^{(2)}
$$

In the rest of this section we estimate each term in (6.18). Remember that $\Lambda=(-L / 2, L / 2)^{\nu} \subset \mathbb{R}^{\nu}$. We first have the following result:

Proposition 6.1. Let $I_{\Lambda, N}$ be defined as in (6.6). There exists a constant c independent of $\Lambda$ and $N$ such that the bound

$$
|\Lambda|^{-1} I_{\Lambda, N} \leq c L^{-1 / 2}
$$

holds.

Proof. (a) The case of the CAR algebra. We use the notation in (6.9)-(6.11). By the bound in (6.8) we have

$$
\begin{aligned}
& I_{\Lambda, N} \leq \sum_{I} \sum_{I^{\prime}:} \omega\left(\sigma_{i / 2}\left(P_{I}\right) P_{I^{\prime}}\right) \\
& I^{\prime} \neq I \\
& =\sum_{\substack{J \subset I(\leq N): \\
J^{c} \neq \emptyset}} \sum_{\substack{I_{1} \in \mathscr{T}(J) \\
I_{2} \in \mathscr{T}\left(J^{c}\right)}} \sum_{I_{2}^{\prime} \in \mathscr{T}\left(J^{c}: I_{2}\right)} \omega\left(\sigma_{i / 2}\left(P_{I_{1}} P_{I_{2}}\right) P_{I_{1}} P_{I_{2}^{\prime}}\right) .
\end{aligned}
$$

Recall the definition of $P_{I}$ in (4.12). Since $J^{c} \neq \emptyset$, there is $k \in J^{c}$ such that

$$
P_{I_{2}}=P_{I_{3}} P_{k}\left(i_{k}\right), \quad P_{I_{2}^{\prime}}=P_{I_{3}^{\prime}}\left(1-P_{k}\left(i_{k}\right)\right)
$$


where $I_{2} \in \mathscr{T}\left(J^{c}-\{k\}\right)$ and $I_{3}^{\prime} \in \mathscr{T}\left(J^{c}-\{k\}: I_{3}\right)$. Thus it follows from (6.19) that

$$
\begin{aligned}
I_{\Lambda, N} \leq & \sum_{\substack{J \subset I(\leq N): \\
J^{c} \neq \emptyset}} \sum_{\substack{I_{1} \in \mathcal{T}(J) \\
I_{3} \in \mathcal{T}\left(J^{c}-\{k\}\right)}} \sum_{\substack { I_{3}^{\prime} \in \mathcal{J}\left(J^{c}-\{k\}: I_{3}\right) \\
\begin{subarray}{c}{k \in J^{c} \\
\imath_{k}=0,1{ I _ { 3 } ^ { \prime } \in \mathcal { J } ( J ^ { c } - \{ k \} : I _ { 3 } ) \\
\begin{subarray} { c } { k \in J ^ { c } \\
\imath _ { k } = 0 , 1 } }\end{subarray}} \sum^{\times\left\{\omega\left(\sigma_{i / 2}\left(P_{I_{1}} P_{I_{3}} P_{k}\left(i_{k}\right)\right) P_{I_{1}} P_{I_{3}^{\prime}}\left(1-P_{k}\left(i_{k}\right)\right)\right)\right\}} \\
\leq & \sum_{k=1}^{N} \sum_{i_{k}=0,1} \sum_{\substack{I \in . \mathcal{T}(I(\leq N)-\{k\}) \\
I^{\prime} \in \mathcal{J}(I(\leq N)-\{k\})}} \omega\left(\sigma_{\imath / 2}\left(P_{I} P_{k}\left(i_{k}\right)\right) P_{I^{\prime}}\left(1-P_{k}\left(i_{k}\right)\right)\right) \\
= & \sum_{k=1}^{N} \sum_{i_{k}=0,1} \omega\left(\sigma_{i / 2}\left(P_{k}\left(i_{k}\right)\right)\left(1-P_{k}\left(i_{k}\right)\right)\right) .
\end{aligned}
$$

We shall use the KMS condition of the following form [3]:

$$
\begin{aligned}
\omega\left(\sigma_{\imath / 2}\left(a^{*}(f) a(g)\right) x\right) & =\omega\left(\sigma_{i / 2}(a(g)) x \sigma_{-i / 2}\left(a^{*}(f)\right)\right) \\
& =\omega\left(a\left(B^{1 / 2} g\right) x a^{*}\left(B^{1 / 2} f\right)\right)
\end{aligned}
$$

for any $f, g \in L^{2}\left(\mathbb{R}^{\nu}\right)$ and $x \in \pi_{\omega}\left(A_{1}\right)^{\prime \prime}$. To obtain the second equality, we have used (4.7) and (4.8). See also Remark 4.2(b).

We recall that $P_{k}(0)=1-N\left(f_{k}\right)=\tilde{N}\left(f_{k}\right), P_{k}(1)=N\left(f_{k}\right)$ and $N\left(f_{k}\right)=$ $a^{*}\left(f_{k}\right) a\left(f_{k}\right)$. Using the KMS condition (6.21), we have

$$
\begin{aligned}
\omega\left(\sigma_{\imath / 2}\left(N\left(f_{k}\right)\right) \tilde{N}\left(f_{k}\right)\right)= & \omega\left(a\left(B^{1 / 2} f_{k}\right) \tilde{N}\left(f_{k}\right) a^{*}\left(B^{1 / 2} f_{k}\right)\right) \\
= & \omega\left(a\left(\left(B^{1 / 2}-\left(B^{\Lambda}\right)^{1 / 2}\right) f_{k}\right) \tilde{N}\left(f_{k}\right) a^{*}\left(B^{1 / 2} f_{k}\right)\right) \\
& +\omega\left(a\left(\left(B^{\Lambda}\right)^{1 / 2} f_{k}\right) \tilde{N}\left(f_{k}\right) a^{*}\left(\left(B^{1 / 2}-\left(B^{\Lambda}\right)^{1 / 2}\right) f_{k}\right)\right) \\
& +\omega\left(a\left(\left(B^{\Lambda}\right)^{1 / 2} f_{k}\right) \tilde{N}\left(f_{k}\right) a^{*}\left(\left(B^{\Lambda}\right)^{1 / 2} f_{k}\right)\right) .
\end{aligned}
$$

Since $a\left(\left(B^{\Lambda}\right)^{1 / 2} f_{k}\right)=\exp \left(-\gamma_{k} / 2\right) a\left(f_{k}\right)$ and $a\left(f_{k}\right)^{2}=0$, the last term in the above equals to zero. Using the fact that $|\omega(A B C)| \leq\|A\|\|B\|\|C\|$, we obtain from the above relation that

$$
\begin{aligned}
& \sum_{k=1}^{N} \omega\left(\sigma_{i / 2}\left(N\left(f_{k}\right)\right) \tilde{N}\left(f_{k}\right)\right) \\
& \quad \leq \sum_{k=1}^{\infty} \|\left(\left(B^{1 / 2}-\left(B^{\Lambda}\right)^{1 / 2}\right) f_{k} \|\left(\left\|B^{1 / 2} f_{k}\right\|+\left\|\left(B^{\Lambda}\right)^{1 / 2} f_{k}\right\|\right)\right. \\
& \quad \leq\left(\operatorname{Tr}\left(\chi_{\Lambda}\left(B^{1 / 2}-\left(B^{\Lambda}\right)^{1 / 2}\right)^{2}\right) \chi_{\Lambda}\right)^{1 / 2} \cdot\left(\operatorname{Tr}(B)^{1 / 2}+\operatorname{Tr}\left(B^{\Lambda}\right)^{1 / 2}\right) \\
& \quad \leq c|\partial \Lambda|^{1 / 2}|\Lambda|^{1 / 2} .
\end{aligned}
$$

Here we have used Lemma 5.2 to obtain the last inequality. Notice that

$$
\omega\left(\sigma_{i / 2}\left(\tilde{N}\left(f_{k}\right)\right) N\left(f_{k}\right)\right)=\omega\left(\sigma_{\imath / 2}\left(N\left(f_{k}\right)\right) \tilde{N}\left(f_{k}\right)\right)
$$

by the KMS condition and so from (6.23) we conclude that

$$
\sum_{k=1}^{N} \omega\left(\sigma_{\imath / 2}\left(\tilde{N}\left(f_{k}\right)\right) N\left(f_{k}\right)\right) \leq c|\partial \Lambda|^{1 / 2}|\Lambda|^{1 / 2} .
$$


Thus the lemma in the case for the CAR algebra follows from (6.20), (6.23) and the above bound.

(b) The case of the CCR algebra: In the case for the CCR algebra, the number operators, $N\left(f_{k}\right), k \in \mathbb{N}$, are not projections and so one has to modify the method used in the proof of the proposition for the CAR algebra so that it can be applicable to the CCR algebra. Notice that the spectral projections $Q_{k}(n)$ of $N\left(f_{k}\right)$ can be expressed as

$$
Q_{k}(n)=\frac{1}{2 \pi i} \oint_{|z|=1 / 2}\left(z-\left(N\left(f_{k}\right)-n\right)\right)^{-1} d z .
$$

Recall that $P_{k}(n)=Q_{k}(n)$ if $n<N$, and $P_{k}(N)=1-\sum_{n=0}^{N-1} Q_{k}(n)$. If one employs the method similar to that used to obtian (6.20), one can obtain the bound

$$
I_{\Lambda, N} \leq \sum_{k=1}^{N} \sum_{l=1}^{N} \sum_{\substack{n=0 \\ n \neq l}}^{N} \omega\left(\sigma_{\imath / 2}\left(P_{k}(l)\right) P_{k}(n)\right) \leq \sum_{k=1}^{N} \sum_{\substack{l, n=0: \\ l \neq n}}^{\infty} \omega\left(\sigma_{\imath / 2}\left(Q_{k}(l)\right) Q_{k}(n)\right) .
$$

We write that

$$
\begin{aligned}
& A_{\Lambda, N}^{(1)}(k)=\sum_{n=0}^{\infty} \omega\left(\sigma_{i / 2}\left(Q_{k}(n+1)\right) Q_{k}(n)\right), \\
& A_{\Lambda, N}^{(2)}(k)=\sum_{n=0}^{\infty} \sum_{l=n+2}^{\infty} \omega\left(\sigma_{i / 2}\left(Q_{k}(l)\right) Q_{k}(n)\right), \\
& A_{\Lambda, N}(k)=A_{\Lambda, N}^{(1)}(k)+A_{\Lambda, N}^{(2)}(k) .
\end{aligned}
$$

It follows from (6.25) and the KMS condition $\omega\left(\sigma_{\imath / 2}(A) B\right)=\omega\left(\sigma_{\imath / 2}(B) A\right)$ that

$$
I_{\Lambda, N} \leq 2 \sum_{k=1}^{N} A_{\Lambda, N}(k)
$$

Let us estimate $A_{\Lambda, N}(k)$. By the CCRs we have that

$$
a\left(f_{k}\right) N\left(f_{k}\right)=\left(N\left(f_{k}\right)+1\right) a\left(f_{k}\right),
$$

and so from (6.24) it follows that

$$
a\left(f_{k}\right) Q_{k}(n)=Q_{k}(n-1) a\left(f_{k}\right) .
$$

Using the above relation and the method employed in (6.22), we obtain

$$
\begin{aligned}
& \omega\left(\sigma_{i / 2}\left(N\left(f_{k}\right) Q_{k}(n+1)\right) Q_{k}(n)\right) \\
& \quad=\omega\left(\sigma_{i / 2}\left(Q_{k}(n)\right) a\left(B^{1 / 2} f_{k}\right) Q_{k}(n) a^{*}\left(B^{1 / 2} f_{k}\right)\right) \\
& \quad=\omega\left(\sigma_{i / 2}\left(Q_{k}(n)\right) a\left(\left(B^{\Lambda}\right)^{1 / 2} f_{k}\right) Q_{k}(n) a^{*}\left(\left(B^{\Lambda}\right)^{1 / 2} f_{k}\right)+Q_{\Lambda, n}^{(1)}(k),\right.
\end{aligned}
$$

where

$$
\begin{aligned}
Q_{\Lambda, n}^{(1)}(k) \equiv & \omega\left(\sigma_{i / 2}\left(Q_{k}(n)\right) a\left(\left(B^{1 / 2}-\left(B^{\Lambda}\right)^{1 / 2}\right) f_{k}\right) Q_{k}(n) a^{*}\left(B^{1 / 2} f_{k}\right)\right) \\
& \left.+\omega\left(\sigma_{i / 2}\left(Q_{k}(n)\right) a\left(\left(B^{\Lambda}\right)^{1 / 2}\right) f_{k}\right) Q_{k}(n) a^{*}\left(\left(B^{1 / 2}-\left(B^{\Lambda}\right)^{1 / 2}\right) f_{k}\right)\right) .
\end{aligned}
$$


Since $N\left(f_{k}\right) Q_{k}(n)=n Q_{k}(n)$ and $\left(B^{\Lambda}\right)^{1 / 2} f_{k}=\exp \left(-\gamma_{k} / 2\right) f_{k}$, it follows from (6.29) and (6.28) that

$$
\begin{aligned}
& \omega\left(\sigma_{i / 2}\left(N\left(f_{k}\right) Q_{k}(n+1)\right) Q_{k}(n)\right) \\
& \quad=\exp \left(-\gamma_{k}\right) n \omega\left(\sigma_{i / 2}\left(Q_{k}(n)\right) Q_{k}(n-1)\right)+Q_{\Lambda, n}^{(1)}(k) .
\end{aligned}
$$

From (6.26) and the above relation, we obtain

$$
\begin{aligned}
A_{\Lambda, n}^{(1)}(k) & \leq \sum_{n=0}^{\infty} \omega\left(\sigma_{i / 2}\left(\left(N\left(f_{k}\right)-n\right) Q_{k}(n+1)\right) Q_{k}(n)\right) \\
& \leq \sum_{n=0}^{\infty}\left\{\exp \left(-\gamma_{k}\right)(n+1)-n\right\} \omega\left(\sigma_{i / 2}\left(Q_{k}(n+1)\right) Q_{k}(n)\right)+\sum_{n=0}^{\infty} Q_{\Lambda, n}^{(1)}(k) \\
& \leq \exp \left(-\gamma_{k}\right) A_{\Lambda, n}^{(1)}(k)+\sum_{n=0}^{\infty} Q_{\Lambda, n}^{(1)}(k)
\end{aligned}
$$

Here we have used the fact that $\exp \left(-\gamma_{k}\right) \leq\left\|B^{\Lambda}\right\|<1$ by (4.8) and (4.6) to get the third inequality. We note that by (4.6), and Assumption 3.1(a) (see Remark 3.2(a))

$$
\begin{aligned}
\left(1-\exp \left(-\gamma_{k}\right)\right)^{-1} & =1+\left\{\exp \left(-\gamma_{k}\right) /\left(1-\exp \left(-\gamma_{k}\right)\right)\right\} \\
& =1+\left(f_{k},\left(B^{\Lambda} /\left(1-B^{\Lambda}\right)\right) f_{k}\right) \\
& =1+\left(f_{k}, A_{\Lambda} f_{k}\right) \\
& \leq 1+M_{1}
\end{aligned}
$$

uniformly in $\Lambda$. Using the above inequality, we obtain from (6.23) that

$$
\sum_{k=1}^{N} A_{\Lambda, n}^{(1)}(k) \leq\left(1+M_{1}\right) \sum_{k=1}^{N} \sum_{n=0}^{\infty} Q_{\Lambda, n}^{(1)}(k) .
$$

We use the Schwarz inequality twice, and the facts that $\left\|Q_{k}(n)\right\|=1$ and $\sum_{n=0}^{\infty} Q_{k}(n)=1$ to obtain from (6.30) and (3.6) that

$$
\begin{aligned}
\sum_{k=1}^{N} \sum_{n=0}^{\infty} Q_{\Lambda, n}^{(1)}(k) \leq & c\left(\operatorname{Tr}\left(\chi_{\Lambda}\left(B^{1 / 2}-\left(B^{\Lambda}\right)^{1 / 2}\right)^{2} \chi_{\Lambda}\right)\right)^{1 / 2} \\
& \times\left\{\left(\operatorname{Tr}\left(B^{\Lambda}\right)\right)^{1 / 2}+\left(\operatorname{Tr}\left(B^{\Lambda}\right)\right)^{1 / 2}\right\}
\end{aligned}
$$

Thus from (6.33), (6.34) and Lemma 5.2, it follows that the bound

$$
\sum_{k=1}^{N} A_{\Lambda, n}^{(1)}(k) \leq c|\partial \Lambda|^{1 / 2}|\Lambda|^{1 / 2}
$$

holds uniformly in $\Lambda$. 
We next consider the term $A_{\Lambda, n}^{(2)}(k)$ defined in (6.26). Using the methods employed in (6.29) and (6.31), one can check that

$$
\begin{aligned}
& \sum_{l=n+2}^{\infty} \omega\left(\sigma_{i / 2}\left(N\left(f_{k}\right) Q_{k}(l)\right) Q_{k}(n)\right) \\
& \quad=\sum_{l=n+2}^{\infty} \omega\left(\sigma_{i / 2}\left(Q_{k}(l-1)\right) a\left(B^{1 / 2} f_{k}\right) Q_{k}(n) a^{*}\left(B^{1 / 2} f_{k}\right)\right) \\
& \quad=\exp \left(-\gamma_{k}\right) \sum_{l=n+2}^{\infty} n \omega\left(\sigma_{\imath / 2}\left(Q_{k}(l-1)\right) Q_{k}(n-1)\right)+B_{\Lambda, n}^{(2)}(k)
\end{aligned}
$$

where

$$
\begin{aligned}
B_{\Lambda, n}^{(2)}(k) \equiv & \sum_{l=n+2}^{\infty} \omega\left(\sigma_{i / 2}\left(Q_{k}(l-1)\right) a\left(\left(B^{1 / 2}-\left(B^{\Lambda}\right)^{1 / 2}\right) f_{k}\right) Q_{k}(n) a^{*}\left(B^{1 / 2} f_{k}\right)\right) \\
& +\sum_{l=n+2}^{\infty} \omega\left(\sigma_{i / 2}\left(Q_{k}(l-1)\right) a\left(\left(B^{\Lambda}\right)^{1 / 2} f_{k}\right) Q_{k}(n)\right. \\
& \left.\times a^{*}\left(\left(B^{1 / 2}-\left(B^{\Lambda}\right)^{1 / 2}\right) f_{k}\right)\right) .
\end{aligned}
$$

Thus from (6.26), (6.36) and the fact that $\exp \left(-\gamma_{k}\right)<1$, we obtain

$$
\begin{aligned}
A_{\Lambda, n}^{(2)}(k) & \leq \sum_{n=0}^{\infty} \sum_{l=n+2}^{\infty} \omega\left(\sigma_{\imath / 2}\left(\left(N\left(f_{k}\right)-(n+1)\right) Q_{k}(l)\right) Q_{k}(n)\right) \\
& \leq \sum_{n=0}^{\infty} B_{\Lambda, n}^{(2)}(k) .
\end{aligned}
$$

We use a method similar to that used to obtain (6.34) to conclude that

$$
\sum_{k=1}^{N} A_{\Lambda, n}^{(2)}(k) \leq c|\partial \Lambda|^{1 / 2}|\Lambda|^{1 / 2}
$$

The proposition follows from (6.27), (6.35) and the above bound. This proves the proposition completely. Q.E.D.

We next consider the term $I I_{\Lambda, N}^{(1.1)}$ defined in (6.17). We first state our result:

Proposition 6.2. There exists a constant $c$ independent of $\Lambda$ and $N$ such that the bound

holds.

$$
|\Lambda|^{-1} I I_{\Lambda, N}^{(1.1)} \leq c|\Lambda|^{-1}
$$

Proof. (a) The case of the CAR algebra: Summing over $P_{I_{1}}$ and using $\omega\left(P_{I_{2}}\right) \leq 1$, one has

$$
I I_{\Lambda, N}^{(1.1)} \leq \sum_{\substack{J \subset I(\leq N): \\ J^{c} \neq \emptyset}} \sum_{I_{2} \in \mathscr{T}\left(J^{c}\right)} \sum_{I_{2}^{\prime} \in \mathscr{T}\left(J^{c}: I_{2}\right)} \omega_{\Lambda, J^{c}}\left(P_{I_{2}}\right) \omega_{\Lambda, J^{c}}\left(P_{I_{2}^{\prime}}\right) .
$$


Recall that $P_{k}(1)=N\left(f_{k}\right)$ and $P_{k}(0)=1-N\left(f_{k}\right)=\tilde{N}\left(f_{k}\right)$ in the case of the CAR algebra. Thus from the definition of $\omega_{\Lambda, J}$ in (6.13), it follows that

$$
\begin{aligned}
I I_{\Lambda, N}^{(1.1)} & \leq \sum_{\substack{J \subset I(\leq N): \\
J^{c} \neq \emptyset}} \prod_{k \in J^{c}}\left\{2 \omega_{\Lambda, J^{c}}\left(N\left(f_{k}\right)\right) \omega_{\Lambda, J^{c}}\left(\tilde{N}\left(f_{k}\right)\right)\right\} \\
& \leq \sum_{J \subset I(\leq N):} \prod_{k \in J^{c}}\left\{2|\Lambda|^{-1} \exp \left(-\gamma_{k}\right) /\left(1+|\Lambda|^{-1} \exp \left(-\gamma_{k}\right)\right)^{2}\right\} \\
& \leq \sum_{J \subset I(\leq N):} \prod_{k \in J^{c}}\left\{2|\Lambda|^{-1} \exp \left(-\gamma_{k}\right)\right\} \\
& =\prod_{k \in I(\leq N):}\left\{1+2|\Lambda|^{-1} \exp \left(-\gamma_{k}\right)\right\} \\
& \leq \exp \left\{\operatorname{Tr} \log \left(1+2|\Lambda|^{-1} B^{\Lambda}\right)\right\} \\
& \leq \exp \left\{c|\Lambda|^{-1} \operatorname{Tr}\left(B^{\Lambda}\right)\right\}
\end{aligned}
$$

for some constant $c$ independent of $\Lambda$ and $N$. The proposition for the CAR algebra now follows from the above bound and Lemma 5.2.

(b) The case of the CCR algebra: From (6.11), (6.13), and (6.38), it follows that

$$
I I_{\Lambda, N}^{(1.1)} \leq \sum_{J \subset I(\leq N):} \prod_{k \in J^{c}}\left\{\sum_{\substack{l, n=0: \\ l \neq n}}^{\infty} \omega_{\Lambda, J^{c}}\left(Q_{k}(l)\right) \omega_{\Lambda, J^{c}}\left(Q_{k}(n)\right)\right\} .
$$

Since for any positive function $f(l, n)$,

$$
\sum_{\substack{l, n=0: \\ l \neq n}}^{\infty} f(l, n) \leq \sum_{l, n=1}^{\infty} f(l, n)+\sum_{l=1}^{\infty} f(l, 0)+\sum_{n=1}^{\infty} f(0, n),
$$

and $\sum_{l=1}^{\infty} Q_{k}(l) \leq N\left(f_{k}\right)$, we obtain from (6.39) that

$$
I I_{\Lambda, N}^{(1.1)} \leq \sum_{J \subset I(\leq N):} \prod_{k \in J^{c}}\left\{\omega_{\Lambda, J^{c}}\left(N\left(f_{k}\right)\right)^{2}\right\}+\sum_{J \subset I(\leq N):} \prod_{k \in J^{c}}\left\{2 \omega_{\Lambda, J^{c}}\left(N\left(f_{k}\right)\right)\right\} .
$$

We now use (6.13) and the fact that $|\Lambda|^{-1} \exp \left(-\gamma_{k}\right) \leq M<1$ uniformly in $\Lambda$ (see the inequality below (6.32)) to conclude that there exists a constant $M$ independent of $\Lambda$ such that

$$
I I_{\Lambda, N}^{(1.1)} \leq \sum_{J \subset I(\leq N):} \prod_{k \in J^{c}}\left(M|\Lambda|^{-1} e^{-\gamma_{k}}\right) \leq \exp \left\{c|\Lambda|^{-1} \operatorname{Tr}\left(B^{\Lambda}\right)\right\} .
$$

Here we have used the method same as that for the CAR algebra to obtain the second inequality above. The proposition follows from Lemma 5.2. This proves the proposition completely. Q.E.D.

Finally we consider the terms $I I_{\Lambda, N}^{(1.2)}$ and $I I_{\Lambda, N}^{(2)}$ defined in (6.17) and (6.14) respectively. We state our result: 
Proposition 6.3. For sufficiently large L, there exists a constant $c$ independent of $\Lambda$ and $N$ such that the bound

holds.

$$
|\Lambda|^{-1}\left(I I_{\Lambda, N}^{(2)}+I I_{\Lambda, N}^{(1.2)}\right) \leq c L^{-1 / 8}
$$

Proof. (a) The case of the CAR algebra: By a symmetry argument $\left(P_{I_{2}} \leftrightarrow P_{I_{2}^{\prime}}\right)$, $I I_{\Lambda, N}^{(1.2)}=I I_{\Lambda, N}^{(2)}$ and so we only need to consider $I I_{\Lambda, N}^{(1.2)}$. Let $P_{I_{2}^{\prime}}$ be of the form

$$
P_{I_{2}^{\prime}}=\left(\prod_{k=0}^{m} N\left(f_{l_{k}}\right)\right)\left(\prod_{k=m+1}^{n} \tilde{N}\left(f_{l_{k}}\right)\right)
$$

where $J^{c}=\left\{l_{1}, l_{2}, \ldots, l_{n}\right\}$. Then from (6.13) it follows that

$$
\omega_{\Lambda, J^{c}}\left(P_{I_{2}^{\prime}}\right)=\prod_{k=0}^{m}\left(|\Lambda|^{-1} \exp \left(-\gamma_{l_{k}}\right)\right) \prod_{k=1}^{n}\left(1+|\Lambda|^{-1} \exp \left(-\gamma_{l_{k}}\right)\right)^{-1}
$$

If $m=0$, then the first factor in the r.h.s. equals to 1 . Thus

$$
\begin{aligned}
-\log \left(\omega_{\Lambda, J^{c}}\left(P_{I_{2}^{\prime}}\right)\right) & =\sum_{k=0}^{m}\left(\gamma_{l_{k}}+\log |\Lambda|\right)+\sum_{k=1}^{n} \log \left(1+|\Lambda|^{-1} \exp \left(-\gamma_{l_{k}}\right)\right) \\
& \leq \sum_{k=0}^{m}\left(\gamma_{l_{k}}+\log |\Lambda|\right)+\operatorname{Tr} \log \left(1+|\Lambda|^{-1} B^{\Lambda}\right)
\end{aligned}
$$

where in the case $m=0$, the first term in the r.h.s. equals to zero. Since $\operatorname{Tr}\left(\log \left(1+|\Lambda|^{-1} B^{\Lambda}\right)\right) \leq c|\Lambda|^{-1} \operatorname{Tr}\left(B^{\Lambda}\right) \leq M$ uniformly in $\Lambda$ by Lemma 5.2, we have

$$
-\log \left(\omega_{\Lambda, J^{c}}\left(P_{I_{2}^{\prime}}\right)\right) \leq \sum_{k=0}^{m}\left(\gamma_{l_{k}}+\log |\Lambda|\right)+M
$$

We substitute the above inequality into $I I_{\Lambda, N}^{(1.2)}$ in (6.17) to obtain that

$$
\begin{aligned}
I I_{\Lambda, N}^{(1.2)} & \left.\leq I I I_{\Lambda, N}+M\left(\sum_{J \subset I(\leq N)} \sum_{\substack{I_{1} \in \mathcal{T}(J) \\
I_{2} \in \mathcal{J}\left(J^{c}\right)}} \omega\left(\sigma_{i / 2}\left(P_{I_{1}} P_{I_{2}}\right) P_{I_{1}} P_{I_{2}^{\prime}}\right)\right)\right) \\
& =I I I_{\Lambda, N}+M\left(\sum_{I, I^{\prime} \in \mathscr{T}(I(\leq N))} \omega\left(\sigma_{i / 2}\left(P_{I}\right) P_{I^{\prime}}\right)\right) \\
& =I I I_{\Lambda, N}+M
\end{aligned}
$$

where

$$
I I I_{\Lambda, N} \equiv \sum_{\substack{J \subset I(\leq N) \\ J^{c} \neq \emptyset}} \sum_{\substack{I_{1} \in \mathscr{T}(J) \\ I_{2} \in \mathscr{T}\left(J^{c}\right)}} \sum_{k=1}^{m} \omega\left(\sigma_{\imath / 2}\left(P_{I_{1}} P_{I_{2}}\right) P_{I_{1}} P_{I_{2}^{\prime}}\right)\left(\gamma_{l_{k}}+\log (|\Lambda|)\right) .
$$

We note that, if $m \neq 0$, there exists $l_{k} \in J^{c}$ such that $P_{I_{2}^{\prime}}$ contains $N\left(f_{l_{k}}\right)$. Thus we may write

$$
P_{I_{2}}=P_{I_{3}} \tilde{N}\left(f_{l_{k}}\right), \quad P_{I_{2}^{\prime}}=P_{I_{3}^{\prime}} N\left(f_{l_{k}}\right)
$$


where $I_{3} \in \mathscr{T}\left(J^{c}-\left\{l_{k}\right\}\right)$ and $\left.I_{3}^{\prime} \in \mathscr{T}\left(J^{c}-\left\{l_{k}\right\}\right): I_{3}\right)$. Notice that $\mathscr{T}\left(J^{c}-\left\{l_{k}\right\}: I_{3}\right)$ is singleton. From (6.41), it follows that

$$
\begin{aligned}
I I I_{\Lambda, N} \leq & \sum_{k=1}^{N} \sum_{J \subset I(\leq N)-\{k\}} \sum_{\substack{I_{1} \in \mathcal{T}(J) \\
I_{3} \in \mathcal{T}\left(J^{c}\right)}} \\
& \times \omega\left(\sigma_{i / 2}\left(P_{I_{1}} P_{I_{3}} \tilde{N}\left(f_{k}\right)\right) P_{I_{1}} P_{I_{3}^{\prime}} N\left(f_{k}\right)\right)\left(\gamma_{k}+\log (|\Lambda|)\right) \\
= & \sum_{k=1}^{N} \sum_{I, I^{\prime} \in \mathcal{T}(I(\leq N)-\{k\})} \omega\left(\sigma_{\imath / 2}\left(P_{I} \tilde{N}\left(f_{k}\right)\right) P_{I^{\prime}} N\left(f_{k}\right)\right)\left(\gamma_{k}+\log (|\Lambda|)\right) \\
= & \sum_{k=1}^{N} \omega\left(\sigma_{\imath / 2}\left(\tilde{N}\left(f_{k}\right)\right) N\left(f_{k}\right)\right)\left(\gamma_{k}+\log (|\Lambda|)\right) \\
= & I I I_{\Lambda, N}^{(1)}+I I I_{\Lambda, N}^{(2)},
\end{aligned}
$$

where

$$
\begin{gathered}
I I I_{\Lambda, N}^{(1)}=\sum_{k=1}^{N} \gamma_{k} \omega\left(\sigma_{i / 2}\left(\tilde{N}\left(f_{k}\right)\right) N\left(f_{k}\right)\right), \\
I I I_{\Lambda, N}^{(2)}=\log (|\Lambda|) \sum_{k=1}^{N} \omega\left(\sigma_{\imath / 2}\left(\tilde{N}\left(f_{k}\right)\right) N\left(f_{k}\right)\right) .
\end{gathered}
$$

Using methods similar to those employed in (6.22) and (6.23), it can be checked that $\omega\left(\sigma_{\imath / 2}\left(\tilde{N}\left(f_{k}\right)\right) N\left(f_{k}\right)\right) \leq\left\|\left(B^{1 / 2}-\left(B^{\Lambda}\right)^{1 / 2}\right) f_{k}\right\|\left(\left\|B^{1 / 2} f_{k}\right\|+\left\|\left(B^{\Lambda}\right)^{1 / 2} f_{k}\right\|\right)$.

Thus it follows from (6.43), (6.44) and Lemma 5.2 that

$$
\begin{aligned}
I I I_{\Lambda, N}^{(2)} \leq & \log (|\Lambda|) \operatorname{Tr}\left(\chi_{\Lambda}\left(B^{1 / 2}-\left(B^{\Lambda}\right)^{1 / 2}\right)^{2} \chi_{\Lambda}\right)^{1 / 2} \\
& \times\left\{\left(\operatorname{Tr}\left(B_{\Lambda}\right)\right)^{1 / 2}+\left(\operatorname{Tr}\left(B^{\Lambda}\right)\right)^{1 / 2}\right\} \\
\leq & 2 \log (|\Lambda|)|\partial \Lambda|^{1 / 2}|\Lambda|^{1 / 2}
\end{aligned}
$$

We next consider $I I I_{\Lambda, N}^{(1)}$ defined in (6.43). Writing $\gamma_{k}=\gamma_{k}^{1 / 4} \gamma_{k}^{3 / 4}$ and using the Schwarz inequality, we obtain

$$
\begin{aligned}
I I I_{\Lambda, N}^{(1)} \leq & \left(\sum_{k=1}^{N} \gamma_{k}^{3 / 2} \omega\left(\sigma_{i / 2}\left(\tilde{N}\left(f_{k}\right)\right) N\left(f_{k}\right)\right)\right)^{1 / 2} \\
& \times\left(\sum_{k=1}^{N} \gamma_{k}^{1 / 2} \omega\left(\sigma_{\imath / 2}\left(\tilde{N}\left(f_{k}\right)\right) N\left(f_{k}\right)\right)\right)^{1 / 2} \\
\leq & \left(\sum_{k=1}^{N} \gamma_{k}^{3 / 2} \omega\left(N\left(f_{k}\right)\right)\right)^{1 / 2}\left(\sum_{k=1}^{N} \gamma_{k} \omega\left(\sigma_{i / 2}\left(\tilde{N}\left(f_{k}\right)\right) N\left(f_{k}\right)\right)\right)^{1 / 4} \\
& \times\left(\sum_{k=1}^{N} \omega\left(\sigma_{\imath / 2}\left(\tilde{N}\left(f_{k}\right)\right) N\left(f_{k}\right)\right)\right)^{1 / 4}
\end{aligned}
$$


We use (6.44) and the fact that $\omega\left(\sigma_{i / 2}\left(\tilde{N}\left(f_{k}\right)\right) N\left(f_{k}\right)\right) \leq \omega\left(N\left(f_{k}\right)\right) \leq \exp \left(-\gamma_{k}\right)$ to obtain from the above bound that

$$
\begin{aligned}
I I I_{\Lambda, N}^{(1)} \leq & \left(\operatorname{Tr}\left(h_{\Lambda}^{3 / 2} B^{\Lambda}\right)\right)^{1 / 2}\left(\operatorname{Tr}\left(h_{\Lambda} B^{\Lambda}\right)\right)^{1 / 4} \\
& \times\left\{\left(\operatorname { T r } ( \chi _ { \Lambda } ( B ^ { 1 / 2 } - ( B ^ { \Lambda } ) ^ { 1 / 2 } ) ^ { 2 } \chi _ { \Lambda } ) ^ { 1 / 8 } \left(\operatorname{Tr}\left(B_{\Lambda}\right)^{1 / 2}+\left(\operatorname{Tr}\left(B^{\Lambda}\right)^{1 / 2}\right\}^{1 / 4}\right.\right.\right. \\
\leq & c|\partial \Lambda|^{1 / 8}|\Lambda|^{7 / 8} .
\end{aligned}
$$

Here we have Lemma 5.2-5.4 to get the last inequality. The proof of proposition follows from (6.40), (6.42), (6.45), and (6.46).

(b) The case of the CCR algebra: As before, we only need to consider $I I_{\Lambda, N}^{(1.2)}$. For given $I_{2}^{\prime}=\left(j_{k}: k \in J^{c}\right) \in \mathscr{T}\left(J^{c}: I_{2}\right), P_{I_{2}^{\prime}}$ has the form

$$
P_{I_{2}^{\prime}}=\prod_{k \in J^{c}} P_{k}\left(j_{k}\right)
$$

Let $\mathscr{H}_{k}$ be the Hilbert space spanned by $\left\{\left(a^{*}\left(f_{k}\right)\right)^{n} \Omega: n=0,1, \ldots\right\}$. One may check that

$$
\omega_{\Lambda, J^{c}}\left(Q_{k}(n)\right)=\operatorname{Tr}_{\mathscr{H}_{k}}\left(\exp \left\{-\left(\gamma_{k}+\log (|\Lambda|) N\left(f_{k}\right)\right\} Q_{k}(n)\right) / N_{\Lambda, k},\right.
$$

where $N_{\Lambda, k}$ is a normalization factor. A direct calculation shows that

$$
\left.\omega_{\Lambda, J^{c}}\left(Q_{k}(n)\right)=\left(1-|\Lambda|^{-1} \exp \left\{-\gamma_{k}\right\}\right)(|\Lambda|)^{-1} \exp \left(-\gamma_{k}\right)\right)^{n}
$$

for any $k \in J^{c}$ and $n=0,1, \ldots$ Notice that by (6.47)

$$
\left.\omega_{\Lambda, J^{c}}\left(P_{k}(N)\right)=\sum_{j=N}^{\infty} \omega_{\Lambda, J^{c}}\left(Q_{k}(j)\right)=(|\Lambda|)^{-1} \exp \left(-\gamma_{k}\right)\right)^{N} .
$$

From (6.47) and (6.48) it follows that

$$
\begin{aligned}
-\log \left\{\omega_{\Lambda, J^{c}}\left(P_{I_{2}^{\prime}}\right)\right\}= & \sum_{k \in J^{c}}-\log \left\{\omega_{\Lambda, J^{c}}\left(P_{k}\left(j_{k}\right)\right)\right\} \\
\leq & \left(\sum_{k \in J^{c}}\left\{-\log \left(1-|\Lambda|^{-1} \exp \left(-\gamma_{k}\right)\right)\right\}\right) \\
& +\left(\sum_{k \in J^{c}} j_{k}\left(\gamma_{k}+\log (|\Lambda|)\right)\right) .
\end{aligned}
$$

Notice that $|\Lambda|^{-1}\left\|B^{\Lambda}\right\| \leq \tilde{M}<1$ for sufficiently large $|\Lambda|$ by (4.6). Since the first term in the r.h.s. of (6.49) is bounded by

$$
\left.\operatorname{Tr}\left(-\log \left\{\left(1-|\Lambda|^{-1} B^{\Lambda}\right)\right\}\right) \leq M^{\prime} \operatorname{Tr}(|\Lambda|)^{-1} B^{\Lambda}\right) \leq M
$$

uniformly in $\Lambda$, we have

$$
-\log \left\{\omega_{\Lambda, J^{c}}\left(P_{I_{2}^{\prime}}\right)\right\} \leq M+\sum_{k \in J^{c}} j_{k}\left(\gamma_{k}+\log (|\Lambda|)\right) .
$$

Substituting (6.50) into $I I_{\Lambda, N}^{(1.2)}$ in (6.17) and employing methods similar to those used to obtain (6.40) and (6.42), it can be shown that

$$
I I_{\Lambda, N}^{(1.2)} \leq \widetilde{I I I}_{\Lambda, N}+M
$$


where

$$
\widetilde{I I I}_{\Lambda, N} \equiv \sum_{k=1}^{N} \sum_{l=0}^{N} \sum_{\substack{n=0 \\ n \neq l}}^{N} n\left(\gamma_{k}+\log (|\Lambda|)\right) \omega\left(\sigma_{\imath / 2}\left(P_{k}(l)\right) P_{k}(n)\right)
$$

It follows from (6.52) and the fact that $\omega\left(\sigma_{i / 2}(A) B\right)=\omega\left(\sigma_{i / 2}(B) A\right)$ that

$$
\begin{aligned}
\widetilde{I I I}_{\Lambda, N} & \leq \sum_{k=1}^{N} \sum_{l=0}^{\infty} \sum_{\substack{n=1: \\
n \neq l}}^{\infty} n\left(\gamma_{k}+\log (|\Lambda|)\right) \omega\left(\sigma_{i / 2}\left(Q_{k}(l)\right) Q_{k}(n)\right) \\
& \leq \sum_{k=1}^{N}\left(\gamma_{k}+\log (|\Lambda|)\right)\left\{\sum_{n<l} l \omega\left(\sigma_{i / 2}\left(Q_{k}(l)\right) Q_{k}(n)\right)\right\} .
\end{aligned}
$$

We now use the method employed in (6.36) to obtain

$$
\begin{aligned}
\sum_{n<l} l \omega\left(\sigma_{i / 2}\left(Q_{k}(l)\right) Q_{k}(n)\right) \leq & \sum_{n<l} \omega\left(\sigma_{i / 2}\left(N\left(f_{k}\right) Q_{k}(l)\right) Q_{k}(n)\right) \\
= & \exp \left(-\gamma_{k}\right) \sum_{n<l} n \omega\left(\sigma_{\imath / 2}\left(Q_{k}(l-1)\right) Q_{k}(n-1)\right) \\
& +\sum_{n=0}^{\infty} Q_{\Lambda, n}(k)
\end{aligned}
$$

where $Q_{\Lambda, n}(k)$ is defined by replacing $\sum_{l=n+2}^{\infty}$ with $\sum_{l=n+1}^{\infty}$ in the definition of $Q_{\Lambda, n}^{(2)}(k)$ in (6.37). The above inequality implies that

$$
\sum_{n<l} l \omega\left(\sigma_{i / 2}\left(Q_{k}(l)\right) Q_{k}(n)\right) \leq\left(1-\exp \left(-\gamma_{k}\right)\right)^{-1} \sum_{n=0}^{\infty} Q_{\Lambda, n}(k) .
$$

We substitute (6.54) into (6.53) and then use the bound at the below of (6.32) to conclude that

$$
\widetilde{I I I}_{\Lambda, N} \leq M^{\prime} \sum_{k=1}^{N}\left(\gamma_{k}+\log (|\Lambda|)\right)\left\{\sum_{n=0}^{\infty} Q_{\Lambda, n}(k)\right\} .
$$

Employing a method similar to that used to obtain (6.34), it can be shown that

$$
\sum_{n=0}^{\infty} Q_{\Lambda, n}(k) \leq M^{\prime \prime}\left\|\left(B^{1 / 2}-\left(B^{\Lambda}\right)^{1 / 2}\right) f_{k}\right\|\left(\left\|B^{1 / 2} f_{k}\right\|+\left\|\left(B^{\Lambda}\right)^{1 / 2} f_{k}\right\|\right) .
$$

By the arguments used in (6.43)-(6.46), one obtains from (6.55) and (6.56) the following bound:

$$
\widetilde{I I I}_{\Lambda, N} \leq c|\partial \Lambda|^{1 / 8}|\Lambda|^{7 / 8} \text {. }
$$

The proposition follows from (6.51) and the above bound. This proved the proposition completely. Q.E.D.

Proof of Proposition 4.3. The proposition follows as a corollary of (6.18) and Proposition 6.1-6.3. Q.E.D. 


\section{Appendix. Estimate of Mean Entropy}

In this appendix we study the thermodynamic limit of the local entropy $S\left(\omega_{\Lambda}\right)$ introduced in (3.20), and produce the proof of Theorem 3.4. We remark that the mean entropy of the Fermi lattice system with respect to quasi-free states have been calculated in [21] by using a method which differs from that in this paper. We first estimate the local entropy.

Proposition A.1. Let $S\left(\omega_{\Lambda}\right)$ be the local entropy defined by (3.20). One has that

$$
S\left(\omega_{\Lambda}\right)=-\operatorname{Tr}\left(A_{\Lambda} \log A_{\Lambda}+\left(1-A_{\Lambda}\right) \log \left(1-A_{\Lambda}\right)\right) \quad(\mathrm{CAR})
$$

and

$$
S\left(\omega_{\Lambda}\right)=-\operatorname{Tr}\left(A_{\Lambda} \log A_{\Lambda}-\left(1+A_{\Lambda}\right) \log \left(1+A_{\Lambda}\right)\right) \quad(\mathrm{CCR})
$$

for any bounded region $\Lambda \subset \mathbb{R}^{\nu}$, where $\operatorname{Tr}$ is the trace over $L^{2}(\Lambda)$. Under Assumption 3.1 (a) and (b), the r.h.s. of the above expressions are finite.

Proof. We first consider the case of the CCR algebra. For any bounded operator $G$ on $L^{2}(\Lambda)$, denote by $\Gamma(G)$ the second quantization of $G$ [3]. Notice that

$$
\Gamma(G) a^{*}(f)=a^{*}(G f) \Gamma(G), \quad f \in L^{2}(\Lambda) .
$$

We assert that the density operator $\varrho_{\Lambda}$ corresponding to $\omega_{\Lambda}$ and the operator $B^{\Lambda}$ defined in (4.6) are related by

$$
\varrho_{\Lambda}=\Gamma\left(B^{\Lambda}\right) / \operatorname{Tr}\left(\Gamma\left(B^{\Lambda}\right)\right)
$$

where $\operatorname{Tr}$ is the trace on $\mathscr{F}_{\Lambda}$.

From the CCRs and (A.1), it follows that

$$
\begin{aligned}
\operatorname{Tr} & \left.\Gamma\left(B^{\Lambda}\right) a^{*}(f) a(g)\right) / \operatorname{Tr}\left(\Gamma\left(B^{\Lambda}\right)\right) \\
& =\operatorname{Tr}\left(a(g) a^{*}\left(B^{\Lambda} f\right) \Gamma\left(B^{\Lambda}\right)\right) / \operatorname{Tr}\left(\Gamma\left(B^{\Lambda}\right)\right) \\
& =\left(g, B^{\Lambda} f\right)+\operatorname{Tr}\left(\Gamma\left(B^{\Lambda}\right) a^{*}\left(B^{\Lambda} f\right) a(g)\right) / \operatorname{Tr}\left(\Gamma\left(B^{\Lambda}\right)\right) .
\end{aligned}
$$

By taking $f=\left(1-B^{\Lambda}\right)^{-1} h$, we have that

$$
\operatorname{Tr}\left(\Gamma\left(B^{\Lambda}\right) a^{*}(h) a(g)\right) / \operatorname{Tr}\left(\Gamma\left(B^{\Lambda}\right)\right)=\left(g,\left(B^{\Lambda} /\left(1-B^{\Lambda}\right)\right) h\right)=\left(g, A_{\Lambda} h\right) .
$$

Since the above relation holds for any $g, h \in L^{2}(\Lambda)$, we proved our assertion. Let $\left\{\lambda_{k}\right\}$ be the eigenvalues of $B^{\Lambda}$ counting multiplicites. Let $\mathscr{T}\left(\mathbb{Z}_{+} ; \mathbb{N}\right)$ be the family of multi-indices $I \in \mathbb{Z}_{+}^{\mathbb{N}}$ which have all but finite number of elements zero. A direct computation shows that [3]

$$
\begin{aligned}
\operatorname{Tr}\left(\Gamma\left(B^{\Lambda}\right)\right) & =\sum_{I \in \mathscr{G}\left(\mathbb{Z}_{+} ; \mathbb{N}\right)} \prod_{m_{k} \in I} \lambda_{k}^{m_{k}} \\
& =\prod_{j}\left(1-\lambda_{j}\right)^{-1} \\
& =\exp \left\{-\operatorname{Tr}_{L^{2}(\Lambda)}\left(\log \left(1-B^{\Lambda}\right)\right)\right\}
\end{aligned}
$$


and

$$
\begin{aligned}
\operatorname{Tr}\left(\Gamma\left(B^{\Lambda}\right) \log \Gamma\left(B^{\Lambda}\right)\right) & =\sum_{I \in \mathscr{T}\left(\mathbb{Z}_{+} ; \mathbb{N}\right)}\left(\prod_{m_{k} \in I} \lambda_{k}^{m_{k}}\right) \log \left(\prod_{m_{k} \in I} \lambda_{k}^{m_{k}}\right) \\
& =\sum_{i=1}^{\infty}\left[\prod_{j \neq \imath}\left(1-\lambda_{j}\right)^{-1}\right]\left(\sum_{m=0}^{\infty} \lambda_{\imath}^{m} \log \lambda_{\imath}^{m}\right) \\
& =\sum_{i=1}^{\infty}\left[\prod_{j \neq i}\left(1-\lambda_{j}\right)^{-1}\right]\left(\log \lambda_{i}\right) \lambda_{i}\left(1-\lambda_{i}\right)^{-2} .
\end{aligned}
$$

Thus it follows from (A.3) and (A.4) that

$$
\begin{aligned}
-\operatorname{Tr}\left(\Gamma\left(B^{\Lambda}\right) \log \Gamma\left(B^{\Lambda}\right)\right) / \operatorname{Tr}\left(\Gamma\left(B^{\Lambda}\right)\right) & =-\sum_{i=1}^{\infty} \frac{\lambda_{i}}{1-\lambda_{\imath}} \log \lambda_{\imath} \\
& =-\operatorname{Tr}_{L^{2}(\Lambda)}\left(\frac{B^{\Lambda}}{1-B^{\Lambda}} \log B^{\Lambda}\right)
\end{aligned}
$$

and so by (A.2) and (A.3)

$$
\begin{aligned}
S\left(\omega_{\Lambda}\right) & =-\operatorname{Tr}\left(\frac{B^{\Lambda}}{1-B^{\Lambda}} \log B^{\Lambda}\right)-\operatorname{Tr}\left(\log \left(1-B^{\Lambda}\right)\right) \\
& =-\operatorname{Tr}\left(A_{\Lambda} \log A_{\Lambda}\right)+\operatorname{Tr}\left(\left(1+A_{\Lambda}\right) \log \left(1+A_{\Lambda}\right)\right) .
\end{aligned}
$$

This gives the expression in the proposition for the CCR algebra. In the case of the CAR algebra one may obtain the corresponding expression by replacing $\mathscr{T}\left(\mathbb{Z}_{+} ; \mathbb{N}\right)$ with $\mathscr{T}(\{0,1\} ; \mathbb{N})$ in (A.3) and (A.4). For the details, see [20].

We next show the finiteness of $S\left(\omega_{\Lambda}\right)$. Consider the case of the CCR algebra. By Assumption 3.1(a) and (4.6), $\left\|B^{\Lambda}\right\| \leq M_{1}<1$ and so

$$
\begin{aligned}
& B^{\Lambda} /\left(1-B^{\Lambda}\right) \leq c_{1} B^{\Lambda}, \\
& -\log \left(1-B^{\Lambda}\right) \leq c_{2} B^{\Lambda}
\end{aligned}
$$

uniformly in $\Lambda$. Notice that $\operatorname{Tr}\left(B^{\Lambda} \log B^{\Lambda}\right)=\operatorname{Tr}\left(h_{\Lambda} B^{\Lambda}\right)$. Thus the finiteness of $S_{\Lambda}(\omega)$ for the CCR algebra follows from (A.5), Lemma 5.2 and Lemma 5.3. The method similar to that used in the above gives the proof for the CAR algebra. This proved the proposition completely. Q.E.D.

We recall the notation in the proof of Lemma 5.3. Let $\Lambda=(-L / 2, L / 2)^{\nu}$ and let $\left\{g_{n}\right\}$ be the orthogonal basis of $L^{2}(\Lambda)$ given by (5.8). Denote by $p_{n}$ the projection operator onto the one-dimensional subspace spanned by the vector $g_{n}$. We introduce an operator $E_{\Lambda}$ on $L^{2}(\Lambda)$ by

$$
E_{\Lambda} \equiv \sum_{n \in \mathbb{Z}^{\nu}} \hat{K}_{A}\left(\frac{2 n \pi}{L}\right) p_{n}
$$

where $\hat{K}_{A}$ is the Fourier transform of $K_{A}$ given in (3.12). Assumption 3.1(a) and (b), and (3.13) imply that there exists a constant $c$ independent of $\Lambda$ such that the bound

$$
\hat{K}_{A}(k) \leq c \prod_{j=1}^{\nu}\left(1+\left|k_{j}\right|\right)^{-(1+\alpha)}
$$


holds. Define

$$
\begin{aligned}
& \tilde{S}\left(\omega_{\Lambda}\right) \equiv-\operatorname{Tr}\left(E_{\Lambda} \log E_{\Lambda}\right)-\operatorname{Tr}\left(\left(1-E_{\Lambda}\right) \log \left(1-E_{\Lambda}\right)\right), \quad(\mathrm{CAR}) \\
& \tilde{S}\left(\omega_{\Lambda}\right) \equiv-\operatorname{Tr}\left(E_{\Lambda} \log E_{\Lambda}\right)+\operatorname{Tr}\left(\left(1+E_{\Lambda}\right) \log \left(1+E_{\Lambda}\right)\right) . \quad(\mathrm{CCR})
\end{aligned}
$$

We then have the following result:

Proposition A.2. For both $\mathrm{CCR}$ and $\mathrm{CAR}$ algebras, the equality

holds.

$$
\lim _{L \rightarrow \infty} \frac{1}{|\Lambda|} S\left(\omega_{\Lambda}\right)=\lim _{L \rightarrow \infty} \frac{1}{|\Lambda|} \tilde{S}\left(\omega_{\Lambda}\right)
$$

The proof of Theorem 3.4 follows as a corollary of the above result:

Proof of Theorem 3.4. Since $K_{B} \in L^{1}\left(\mathbb{R}^{\nu}\right)$, Assumption 3.1(a) and (3.13) imply that $\hat{K}_{A}$ is continuous. Notice that

$$
-\operatorname{Tr}\left(E_{\Lambda} \log E_{\Lambda}\right)=-\sum_{n \in \mathbb{Z}^{\nu}} \hat{K}_{A}(2 n \pi / L) \log \left(\hat{K}_{A}(2 n \pi / L)\right) .
$$

Using the continuity of $\hat{K}_{A}$ and the bound in (A.7), one concludes that

$$
-\frac{1}{|\Lambda|} \operatorname{Tr}\left(E_{\Lambda} \log E_{\Lambda}\right) \rightarrow-\frac{1}{(2 \pi)^{\nu}} \int \hat{K}_{A}(k) \log \hat{K}_{A}(k) d^{\nu} k
$$

as $L \rightarrow \infty$. The second terms in (A.8) give the corresponding second terms in Theorem 3.4 Q.E.D.

The rest of this paper is devoted to the proof of Proposition A.2.

Proof of Proposition A.2. We first consider $-\operatorname{Tr}\left(A_{\Lambda} \log A_{\Lambda}\right)$. Let $D_{\Lambda}$ be the operator on $L^{2}(\Lambda)$ defined in (5.10). Let $a(L)$ be a positive number satisfying

$$
\lim _{L \rightarrow \infty} a(L)=0 .
$$

We shall specify the number $a(L)$ later. For $0<2 \alpha^{\prime}<\alpha<1$, denote

$$
F_{\Lambda} \equiv E_{\Lambda}+a(L) D_{\Lambda} \text {. }
$$

Notice that

$$
\begin{aligned}
- & \left\{\operatorname{Tr}\left(A_{\Lambda} \log A_{\Lambda}\right)-\operatorname{Tr}\left(E_{\Lambda} \log E_{\Lambda}\right)\right\} \\
= & -\operatorname{Tr}\left(A_{\Lambda} \log A_{\Lambda}-A_{\Lambda} \log F_{\Lambda}\right)-\operatorname{Tr}\left(\left(A_{\Lambda}-F_{\Lambda}\right) \log F_{\Lambda}\right) \\
& -\left\{\operatorname{Tr}\left(F_{\Lambda} \log F_{\Lambda}\right)-\operatorname{Tr}\left(E_{\Lambda} \log E_{\Lambda}\right)\right\} \\
\equiv & I_{\Lambda}+I I_{\Lambda}+I I I_{\Lambda} .
\end{aligned}
$$

We assert that

$$
|\Lambda|^{-1}\left(\left|I_{\Lambda}\right|+\left|I I_{\Lambda}\right|+\left|I I I_{\Lambda}\right|\right) \rightarrow 0
$$

as $L \rightarrow \infty$. Under the assertion we conclude that

$$
\lim _{L \rightarrow \infty}|\Lambda|^{-1}\left(-\operatorname{Tr}\left(A_{\Lambda} \log A_{\Lambda}\right)\right)=\lim _{L \rightarrow \infty}|\Lambda|^{-1}\left(-\operatorname{Tr}\left(E_{\Lambda} \log E_{\Lambda}\right)\right) .
$$

We prove our assertion in (A.12). Using inequality (4.27), we obtain

$$
\begin{aligned}
\left|I_{\Lambda}\right| & \leq\left|\operatorname{Tr}\left(F_{\Lambda}-A_{\Lambda}\right)\right| \\
& =\left|\operatorname{Tr}\left(E_{\Lambda}+a(L) D_{\Lambda}-A_{\Lambda}\right)\right| .
\end{aligned}
$$


Since $|\Lambda|^{-1}\left|\operatorname{Tr}\left(E_{\Lambda}-A_{\Lambda}\right)\right| \rightarrow 0$ and $a(L)|\Lambda|^{-1} \operatorname{Tr}\left(D_{\Lambda}\right) \rightarrow 0$ as $L \rightarrow \infty$, we conclude that $|\Lambda|^{-1}\left|I_{\Lambda}\right| \rightarrow 0$ as $L \rightarrow \infty$. Similarly it is easy to show that $|\Lambda|^{-1}\left|I I I_{\Lambda}\right| \rightarrow 0$ as $L \rightarrow \infty$.

We next consider $I I_{\Lambda}$. Notice that if $\left(g_{n},\left(E_{\Lambda}+a(L) D_{\Lambda}\right) g_{n}\right) \leq 1$,

$$
\left|\left(g_{n}, \log \left(E_{\Lambda}+a(L) D_{\Lambda}\right) g_{n}\right)\right| \leq\left|\left(g_{n}, \log \left(a(L) D_{\Lambda}\right) g_{n}\right)\right|
$$

and if $\left(g_{n},\left(E_{\Lambda}+a(L) D_{\Lambda}\right) g_{n}\right) \geq 1$,

$$
\left|\left(g_{n}, \log \left(E_{\Lambda}+a(L) D_{\Lambda}\right) g_{n}\right)\right| \leq \mathrm{const}
$$

uniformly in $\Lambda$ by Assumption 3.1(a), (3.14) and (A.6). Thus we have that

$$
\begin{aligned}
|\Lambda|^{-1}\left|I I_{\Lambda}\right| \leq & |\Lambda|^{-1} \sum_{n \in \mathbb{Z}^{\nu}}\left|\left(g_{n},\left(A_{\Lambda}-E_{\Lambda}\right) g_{n}\right)\right||2 \pi n / L|^{2 \alpha^{\prime}} \\
& -\log (a(L))|\Lambda|^{-1} \mid \operatorname{Tr}\left(\left(A_{\Lambda}-E_{\Lambda}\right) \mid\right. \\
& +a(L)|\Lambda|^{-1}\left|\operatorname{Tr}\left(D_{\Lambda} \log \left(a(L) D_{\Lambda}\right)\right)\right| \\
\equiv & b_{1}(L)+b_{2}(L)+b_{3}(L)
\end{aligned}
$$

Since $a(L) \rightarrow 0$ as $L \rightarrow \infty$, a direct computation shows that $b_{3}(L) \rightarrow 0$ as $L \rightarrow \infty$. We now choose the number $a(L)$ as $a(L)=|\Lambda|^{-1}\left|\operatorname{Tr}\left(A_{\Lambda}-E_{\Lambda}\right)\right|$. Then $a(L) \rightarrow 0$ as $L \rightarrow \infty$, and so $b_{2}(L) \rightarrow 0$ as $L \rightarrow \infty$. The term $b_{1}(L)$ defined in (A.14) can be written as

$$
b_{1}(L)=\left.|\Lambda|^{-1} \sum_{n \in \mathbb{Z}^{\nu}}\left|\frac{2 n \pi}{L}\right|^{2 \alpha^{\prime}}\left|\int\right| \hat{\chi}_{\Lambda, n}(k)\right|^{2} \hat{K}(k) d^{\nu} k-\hat{K}\left(\frac{2 n \pi}{L}\right) \mid,
$$

where $\hat{\chi}_{\Lambda, n}$ has been defined in (5.13). Change of variables yields that

$$
\begin{aligned}
\hat{K}_{L, n} & \equiv \int\left|\hat{\chi}_{\Lambda, n}(k)\right|^{2} \hat{K}_{A}(k) d^{\nu} k \\
& =\pi^{-\nu} \int\left(\prod_{j=1}^{\nu} \sin ^{2}\left(k_{j}\right) / k_{j}^{2}\right) \hat{K}_{A}((2 k / L)+(2 \pi n / L)) d^{\nu} k .
\end{aligned}
$$

Notice that $\pi^{-1} \int\left(\sin ^{2} k\right) k^{-2} d k=1$. For given $k^{\prime} \in R^{\nu}$, take $L \rightarrow \infty$ and $|n| \rightarrow \infty$ such that $\lim (2 n \pi / L)=k^{\prime}$. Then the dominated convergence theorem implies that $\hat{K}_{L, n} \rightarrow \hat{K}_{A}\left(k^{\prime}\right)$. If one uses the bound (A.7), $2 \alpha^{\prime}<\alpha$ and the dominated convergence theorem, one can show that $b_{1}(L) \rightarrow 0$ as $L \rightarrow 0$. We leave the details to the reader. Thus from (A.14) and the above results it follows that $|\Lambda|^{-1}\left|I I_{\Lambda}\right| \rightarrow 0$ as $L \rightarrow 0$. This proved (A.12) completely.

It is clear that a straightforward application of the method used in the proof of (A.13) shows that

$$
|\Lambda|^{-1} \mid \operatorname{Tr}\left(\left(1 \pm A_{\Lambda}\right) \log \left(1 \pm A_{\Lambda}\right)-\operatorname{Tr}\left(\left(1 \pm E_{\Lambda} \log \left(1 \pm E_{\Lambda}\right)\right) \mid \rightarrow 0\right.\right.
$$

as $L \rightarrow \infty$. We again leave the detailed proof of the above result to the reader. This completes the proof of Proposition A.2. Q.E.D. 


\section{References}

1. Accardi, L., Cecchini, C.: Conditional expectations in von Neumann algebras and a theorem of Takesaki, J. Funct. Anal. 45, 245-273 (1982)

2. Araki, H., Shiraishi, M.: On quasi-free states of the canonical commutation relations I. Publ. R.I.M.S., Kyoto UNiv. 7, 105-120 (1971)

3. Bratteli, O., Robinson, D.W.: Operator algebras and quantum statistical mechanics, Vols. I, II. Berlin, Heidelberg, New York: Springer 1979

4. Choi, M.D., Effros, E.G.: Ann. Math. 104, 585 (1976)

5. Confeld, I.P., Fomin, S.V., Sinai, Ya.G.: Ergodic theory. Berlin, Heidelberg, New York: Springer 1980

6. Connes, A., Störmer, E.: Acta. Math. 134, 289 (1975)

7. Connes, A., Narnhofer, H., Thirring, W.: Dynamical entropy of $C^{*}$-algebras and von Neumann algebras. Commun. Math. Phys. 112, 691 (1987)

8. Kadison, R.V., Ringrose, J.R.: Fundamentals of the theory of operator algebras, Vols. I, II. New York: Academic Press 1986

9. Kolmogorov, A.N.: Dokl. Akad. Nauk. 119, 861 (1958)

10. Narnhofer, H.: Quantized Arnold cat maps can be entropic $K$ systems. UWThPh (1991)

11. Narnhofer, H., Thirring, W.: Dynamical entropy and the third law of thermodynamics. Lett. Math. Phys. 15, 261-273 (1988)

12. Narnhofer, H., Thirring, W.: Quantum $K$-systems. Commun. Math. Phys. 125, 565-577 (1989)

13. Narnhofer, H., Thirring, W.: Chaotic properties of the noncommutative 2-shift. UWThPh (1991)

14. Park, Y.M.: Quantum statistical mechanics for superstable interactions; Bose-Einstein statistics. J. Stat. Phys. 40, 259 (1984)

15. Park, Y.M.: Quantum statistical mechanics of unbounded continuous spin systems. J. Korean Math. Soc. 22.1, 43-74 (1985)

16. Park, Y.M., Shin, H.H.: Dynamical entropy of quasi-local algebras in quantum statistical mechanics. Commun. Math. Phys. 144, 149-161 (1992)

17. Ruelle, D.: Statistical Mechanics. New York: Benjamin Inc. 1978

18. Sinai, Ya.G.: Dokl. Akad. Nauk. 124, 768 (1959)

19. Störmer, E., Voiculescu, D.: Entropy of Bogoliubov Automorphisms of Canonical Anticommutation Relations. Commun. Math. Phys. 133, 521-542 (1990)

20. Verbeure, A.: Normal and locally normal quasi-free states of Fermi systems. In Cargése Lecture in Physics, Vol. 4 (1970)

21. Fannes, M.: The entropy density of Quasi-free states. Commun. Math. Phys. 31, 279-290 (1973)

22. Herman, R.H., Takesaki, M.: States and automorphism groups of operator algebras, Commun. Math. Phys. 19, 142-160 (1970)

Note added in proof. After making the preprint of this paper we received the following preprint: 23. Narnhofer, H., Thirring, W.: Dynamical Entropy of Quantum Systems and Their Abelian Counterpart. UWYhPh (1991)

which contains the result similar to that of the CAR algebras in this paper. However the method employed in [21] is the momentum space analysis which differs to the position space analysis we used. 
